NISSUNA UMANA INVESTIGAZIONE SI PUO DIMANDARE VERA SCIENZIA S'ESSA NON PASSA PER LE MATEMATICHE DIMOSTRAZIONI LEONARDO DA VINCI

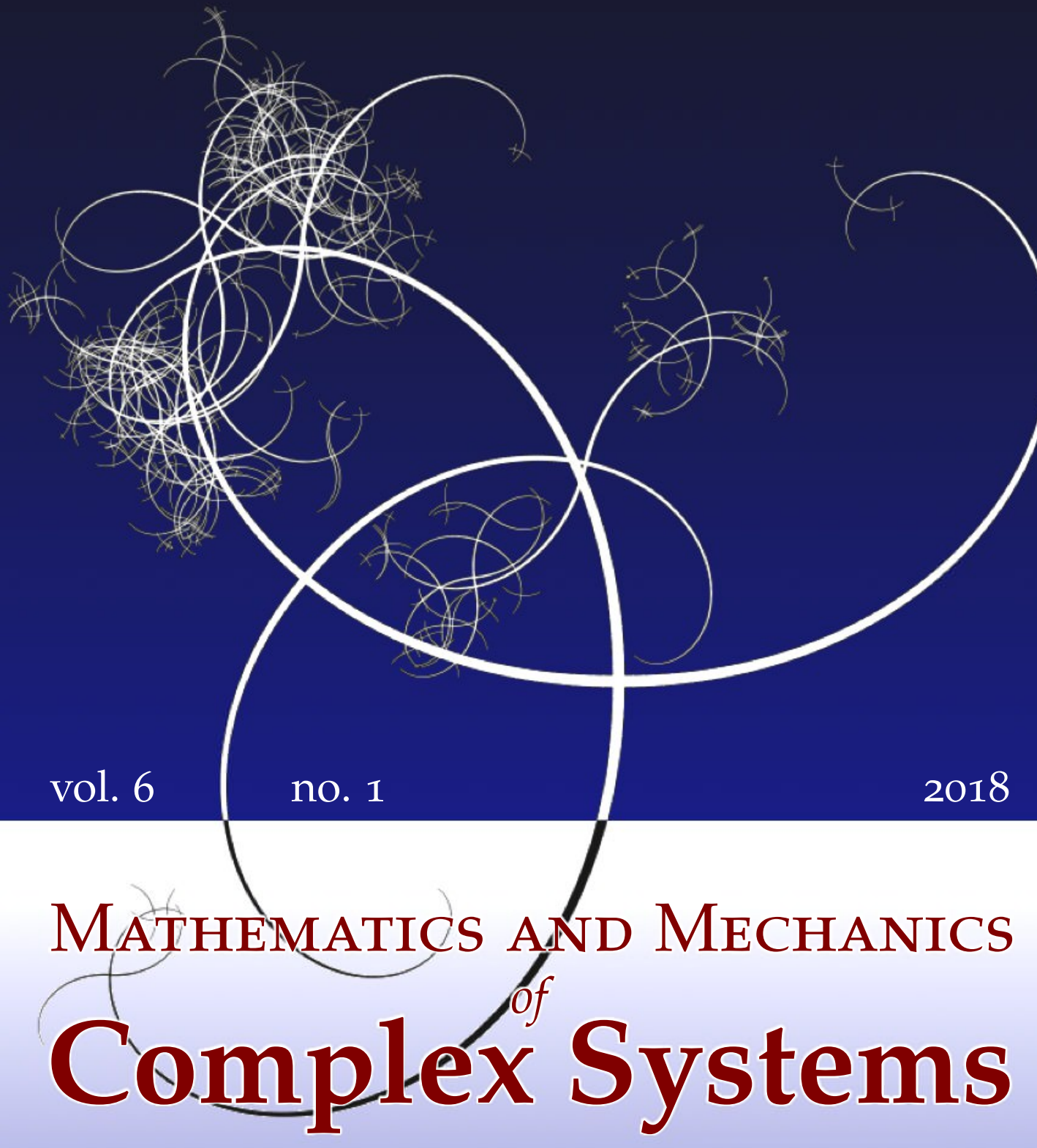

WOLFGANG H. MÜLLER

THE EFFECT OF ROTATION

ON THE FLATTENING OF CELESTIAL BODIES:

A JOURNEY THROUGH FOUR CENTURIES 


\title{
THE EFFECT OF ROTATION \\ ON THE FLATTENING OF CELESTIAL BODIES: A JOURNEY THROUGH FOUR CENTURIES
}

\author{
WOLFGANG H. MÜLleR
}

\begin{abstract}
This paper presents an overview and comments on various continuum models used for predicting the deformation of celestial objects under their own rotation, also known as "flattening" — in particular from a historical perspective. Initially we shall discuss the chronology of events leading to models for fluids, solids, and gases. Our review will range from Newton's famous Principia, Thomson and Tait's Treatise on natural philosophy, and the treatise of the spinning top by Klein and Sommerfeld to the modern literature, which accounts for quantum mechanics and relativistic effects in exotic spinning celestial objects, such as neutron stars and white dwarfs. Then, based on previously published results by Müller and Lofink (2014) and Müller and Weiss (2016), we will present a modern treatment of the fluid model according to Newton. It will be applied not only to the Earth but also to other celestial bodies. We will compare the results to actual measurements and discuss reasons for discrepancies. Finally, we turn to a model for a solid based on Hookean linear elasticity, which we shall also state and solve in modern terminology. In particular, we will not only compute the flattening but also present closed-form solutions for the stresses in a gravitating and stationary spinning, linear-elastic sphere.
\end{abstract}

\section{Some historical background and the experimental evidence}

We all know that the rotational movement of matter against an inertial system gives rise to centrifugal accelerations, leading first to complex motion, which then eventually ceases and results in stationary deformation. In particular, if the rotating matter is a gas or a liquid, these deformations may be considerable. Indeed, the great Newton [1972, p. 51] himself describes this effect in words in his famous bucket experiment, i.e., the formation of what we now know to be a parabolic free water surface: “... superficies aquae sub initio plana erit, quemadmodum ante

\section{Communicated by Francesco dell'Isola.}

MSC2010: 85-03, 85A99, 31-00, 74B05, 83-03.

Keywords: flattening, history of planet flattening, stresses in celestial bodies, fluid, degenerate matter, barytropic equations of state, linear-elastic Hookean model of Earth, linear elasticity, hydrostatics, self-gravitation. 

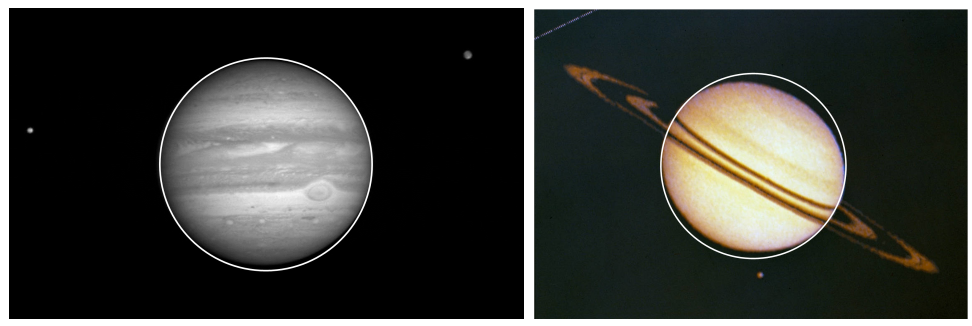

Figure 1. Photographs showing the optically visible, yet small flattening of Jupiter and Saturn. For convenience circles illustrating a perfect spherical shape have been added [Wikipedia 2016b; NASA 2017].

motum vasis, at postquam, vi in aquam paulatim impressa, effecit vas, ut haec quoq; sensibiliter revolvi incipiat, recedet ipsa paulatim a medio, ascendetque ad latera vasis, figuram concavam induens, (ut ipse expertus sum)..."1 Note that his description is purely qualitative as well as heuristic, namely based on experience, as he says himself, despite the fact that he had developed calculus and was most likely in a position to predict the shape of the free surface mathematically. However, no such attempt is made, at least not in his Principia.

The incompressible fluid model. This is different in the case of predicting the figure of the Earth and other planets. Newton specifically mentions Jupiter showing a huge amount of flattening, which was easily observable, even in the old days, as well as the Earth; see Figure 1. He idealizes both planets as fluid bodies and says in [Newton 1972, p. 592], "Planetae sublato omni motu circulari diurno figuram sphaericam, ob aequalem undique partium gravitatem, affectare deberent. Per motum illum circularem sit ut partes ab axe recedentes juxta aequatorem ascendere conentur. Ideoque materia si fluida sit ascensu suo ad aequatorem diametros adaugebit, axem vero descensu suo ad polos diminuet. Sic jovis diameter (consentientibus astronomorum observationibus) brevior deprehenditur inter polos quam $\mathrm{ab}$ oriente in occidentem. Eodem argumento, nisi terra nostra paulo altior esset sub aequatore quam ad polos, maria ad polos subsiderent, \& juxta aequatorem

\footnotetext{
${ }^{1}$ Unfortunately translations of the original Latin text are never word by word and hence distract from the original power of Newton's thoughts and sometimes cloud what he really meant. Also, be advised that Newton's Principia come in various editions with substantial amendments from version to version. A true scholar of the history of science would point out and study the differences. For brevity we are not going to attempt this here. Nevertheless, the reader who wishes to consult a (nonverbatim) translation of the original may want to turn to [Newton 1999, p. 81]: “... the surface of the water will at first be plain, as before the vessel began to move; but the vessel, by gradually communicating its motion to the water, will make it begin sensibly to revolve, and recede little by little from the middle, and ascend to the sides of the vessel, forming itself into a concave figure (as I have experienced)..."
} 
ascendendo, ibi omnia inundarent." ${ }^{2}$ The latter is obviously a gut feeling statement of Newton, the discoverer of the law of gravity, as we shall see shortly.

Indeed, the rest of the passage anticipates the mathematical definition of the flattening or (more precisely) the ellipticity, $f$, which is given by the ratio [Stacey and Davis 2008, Chapter 6]

$$
f=\frac{a-c}{a}
$$

where $a$ denotes the (mean) equatorial radius and $c$ the polar radius of a celestial body. In contrast to the bucket problem Newton [1972, p. 595] quantifies the flattening of the Earth, first, based on geodesic experiments of his time: “...Vis centrifuga corporum in aequatore terrae est ad vim centrifugam, qua corpora directe tendunt a terra in latitudine Lutetiae graduum $48^{\circ} 50^{\prime} 10^{\prime \prime}$, in duplicata ratione radii ad sinum complementi latitudinis illius, id est, ut 7,54064 ad 3,267. Addatur haec vis ad vim qua gravia descendunt in latitudine illa Lutetiae, \& corpus in latitudine illa, vi tota gravitatis cadendo, tempore minuti unius secundi describet lineas 2177,267 seu pedes Parisienses 15 dig. $1 \&$ lin. 5,267. Et vis tota gravitatis in latitudine illa erit ad vim centrifugam corporum in aequatore terrae ut 2177,267 ad 7,54064 seu 289 ad 1."3

Second, Newton conceives a rather strange fluid model of the Earth, which Chandrasekhar [1995, p. 384] calls "method of the canals": two straight canals (see Figure 2), one along the equatorial and one along the polar axis of the Earth, are filled with water and interconnected at a right angle. Newton considers stationary conditions and equilibrium of forces resulting from gravity and centrifugal acceleration. However, he does not really detail the mathematical analysis. Rather he explains his findings by many words: Propositio XIX, Problema III and Propositio XX, Problema IV of [Newton 1999, p. 593]. Another excerpt may suffice

2 "The equal gravitation of the parts on all sides would give a spherical figure to the planets, if it was not for their diurnal revolution in a circle. By that circular motion it comes to pass that the parts receding from the axis endeavour to ascend about the equator; and therefore if the matter is in a fluid state, by its ascent towards the equator it will enlarge the diameters there, and by its descent towards the poles it will shorten the axis. So the diameter of Jupiter (by the concurring observations of astronomers) is found shorter betwixt pole and pole than from east to west. And, by the same argument, if our earth was not higher about the equator than at the poles, the seas would subside about the poles, and, rising towards the equator, would lay all things there under water" [Newton 1999, p. 405].

3 "The centrifugal force of bodies in the equator is to the centrifugal force with which bodies recede directly from the earth in the latitude of Paris $48^{\circ} 50^{\prime} 10^{\prime \prime}$ in the duplicate proportion of the radius to the cosine of the latitude, that is, as 7,54064 to 3,267. Add this force to the force with which bodies descend by their weight in the latitude of Paris, and a body, in the latitude of Paris, falling by its whole undiminished force of gravity, in the time of one second, will describe 2177,267 lines, or 15 Paris feet, 1 inch, and 5,267 lines. And the total force of gravity in that latitude will be to the centrifugal force of bodies in the equator of the earth as 2177,267 to 7,54064 , or as 289 to 1 ", [Newton 1999, p. 106]. 


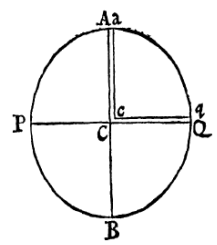

Figure 2. A sketch from the Principia to illustrate Newton's method of canals [1972, p. 596]. Note that strangely enough Newton's equator runs from $A$ to $B$, i.e., from top to bottom.

in order to illustrate the point: "Quoniam pondera inaequalium crurum canalis aqueae $A C Q q c a$ aequalia sunt; \& pondera partium, cruribus totis proportionalium \& similiter in totis sitarum, sunt ad invicem ut pondera totorum, iadeoque etiam aequantur inter se; erunt pondera aequalium $\&$ in cruribus similiter sitarum partium reciproce ut crura, id est reciproce ut 230 ad 229. Et par est ratio homogeneorum $\&$ aequalium quorumvis $\&$ in canalis cruribus similiter sitorum corporum. Horum pondera sunt reciproce ut crura, id est, reciproce ut distantiae corporum a centro terrae. Proinde si corpora in supremis canalium partibus, sive in superficie terrae consistant; erunt pondera eorum ad invicem reciproce ut distantiae eorum a centro. Et eodem argumento pondera, in aliis quibuscunque per totam terrae superficiem regionibus, sunt reciproce ut distantiae locorum a centro; $\&$ propterea, ex hypothesi quod terra sphaerois sit, dantur proportione."4

Luckily, we have Chandrasekhar who translates Newton's thoughts for us and explains what all of this means, in particular all the data and numbers that are mentioned by Sir Isaac. Chandrasekhar [1995, p. 384] derives the following formula, which, as we shall see shortly, is valid for small values of $f$ :

$$
f=\frac{5}{4} \frac{a \omega_{0}^{2}}{G m / a^{2}}=\frac{5}{4} \frac{\omega_{0}^{2} a^{3}}{G m}=\frac{5}{4} \frac{3 \omega_{0}^{2}}{4 \pi G \rho_{0}},
$$

where $G=6.673 \cdot 10^{-11} \frac{\mathrm{m}^{3}}{\mathrm{~kg} \cdot \mathrm{s}^{2}}$ stands for the gravitational constant, $m$ is the mass of the celestial body, and $\omega_{0}$ is its (constant) angular velocity. The last way of writing

4"Because the weights of the unequal legs of the canal of water ACQqca are equal; and the weights of the parts proportional to the whole legs, and alike situated in them, are one to another as the weights of the wholes, and therefore equal betwixt themselves; the weights of equal parts, and alike situated in the legs, will be reciprocally as the legs, that is, reciprocally as 230 to 229 . And the case is the same in all homogeneous equal bodies alike situated in the legs of the canal. Their weights are reciprocally as the legs, that is, reciprocally as the distances of the bodies from the centre of the earth. Therefore if the bodies are situated in the uppermost parts of the canals, or on the surface of the earth, their weights will be one to another reciprocally as their distances from the centre. And; by the same argument, the weights in all other places round the whole surface of the earth are reciprocally as the distances of the places from the centre; and, therefore, in the hypothesis of the earth being a spheroid are given in proportion" [Newton 1999, p. 409]. 
the formula emphasizes incompressibility; i.e., a constant mass density, $\rho_{0}$, was used. Thus, we may suggestively say that the flattening is basically given by the ratio of angular acceleration to gravitational acceleration. The ominous factor $\frac{5}{4}$ accounts for the difference between the gravitational acceleration at the pole and at the equator, which follows from the specific weights of the two water columns in an oblate spheroid. Further below we shall derive this result slightly differently than Chandrasekhar did and show precisely how the factor originates.

It is more than fair to point out that Newton withheld many details explaining how his law of gravity would lead to this result. In fact, Chandrasekhar has good reasons to believe that Newton knew the formula for the gravitational attraction of an oblate spheroid, which we shall use further below as well. Needless to say that Newton also does not provide a concise formula for the flattening, such as the one above. Rather his approach is a mix of (hidden) theory and experimental evidence as we tried to demonstrate by the previous two citations from the Principia. At the end of his gedanken experiment with the two interconnected canals, and after a long line of arguments involving equilibrium of forces and his law of gravity, he concludes that the flattening of the Earth is given as, "Et altitudo ejus ad aequatorem erit 19658600 pedum circiter, \& ad polos 19573000 pedum" [Newton 1972, p. 598]..$^{5}$

Chandrasekhar [1995, p. 389] presents a translation of Newton's line of thoughts together with many annotations. We leave it to the reader to study the details and conclude only that Newton's predicted value for the flattening of a homogeneous Earth is $f_{E}=4.35 \cdot 10^{-3}$. Furthermore, if we take (1-2) for granted and insert the currently accepted values for the mass of the Earth, $m_{E}=5.972 \cdot 10^{24} \mathrm{~kg}$, its equatorial radius, $a_{E}=6.378 \cdot 10^{6} \mathrm{~m}$, and its angular (sidereal) speed $\omega_{0, E}=$ $2 \pi / 86164 \mathrm{~s}$ [Wikipedia 2016a; 2016c], we find that $f_{E}=4.33 \cdot 10^{-3}$. Indeed, this is amazingly close to Newton's figure. However, modern sources quote a different number for the observed flattening of the Earth, e.g., NASA's $f_{E}=3.35 \cdot 10^{-3}$ [Williams 2017].

Why the discrepancy? As we shall see below, one of the key factors is the choice of the "correct" constitutive equation, for example, an incompressible fluid model in the present case, which forms the basis of (1-2). Moreover, in the same context a remark is in order: frequently it remains unclear as to whether a quoted number for the flattening truly results from direct length measurements or from some model equation. In other words, circular conclusions are immanent (also see [Bridgman 1958] regarding the interrelation of physical theory and experiment). We will comment some more on this issue later. Furthermore, it is also known that Earth is not homogeneous and the denser, heavier matter is at the center. This will

5"And its height at the equator will be about 19658600 feet, and at the poles 19573000 feet" [Newton 1999, p. 108]. 
also affect the flattening. By looking at (1-2) one may say with a grain of salt that the effective density, $\rho_{0}$, increases. Hence, the flattening decreases.

It is worth mentioning that Newton also applies his fluid model to explain the rather large flattening value, $f_{J}$, for Jupiter. For this purpose, he reinterprets (1-2) in terms of (homogeneous, constant) mass densities, $\rho$, so to speak:

$$
f=\frac{3}{4 \pi} \frac{\omega_{0}^{2}}{G \rho} \Rightarrow f_{J}=f_{E}\left(\frac{\omega_{0, J}}{\omega_{0, E}}\right)^{2} \frac{\rho_{E}}{\rho_{J}} .
$$

Newton had some information on the relative densities of celestial bodies based on his gravitational law, the measured times of revolution, and the distances of the moons orbiting around them: "Erant autem verae solis, jovis, saturni ac terrae diametri ad invicem ut $10000,997,791, \& 109, \ldots \&$ propterea densitates sunt ut 100, 94 $\frac{1}{2}, 67 \& 400$ " [Newton 1972, Liber III, Propositio VIII, Theorema VIII, Corol 3 on p. 582]. ${ }^{6}$ He concludes: “... sintque temporum quadrata ut 29 ad 5, \& revolventium densitates ut 400 ad $94 \frac{1}{2}$ (see $\left.(1-3)_{2}\right)$... Est igitur diameter iovis ab oriente in occidentem ducta, ad ejus diametrum inter polos ut $10 \frac{1}{3}$ ad $9 \frac{1}{3}$ quamproxime" [Newton 1972, p. 599]. ${ }^{7}$ We conclude that $f_{J}=9.68 \cdot 10^{-2}$. However, the modern figure reported by NASA is $f_{J}=6.49 \cdot 10^{-2}$ [Williams 2017]. It is curious to note that in both cases, for the Earth as well as for Jupiter, the predicted flattening value is higher than the actually observed one. In contrast to that the simplest solid model leads to an underestimate, as we shall see now.

The Hookean model. We shall now consider the historical development of solid mechanics models for the flattening. First, let us ask why we need such models at all? For an answer we simply quote from [Klein and Sommerfeld 2012, p. 687]: "...the assumption of a fluid interior in a compliant shell is refuted by the phenomenon of the tides. A thin crust of the Earth with the elastic compliability of the materials known to us would follow the deforming influence of the tidal forces almost as willingly as the water of the sea. There would then be, however, no relative motion of the water with respect to the land under the influence of these forces, but only a common rise and fall of the sea and the continents that would escape immediate perception. Thus there remains only the assumption that the Earth is, in the mean, effectively solid...."

Note that the emphasis in this citation is on tidal effects. Consequently, it is not surprising that the flattening problem is frequently discussed in context with the

6 "But the true diameters of the Sun, Jupiter, Saturn, and the Earth, were one to another as 10000 , 997, 791, and 109; and the weights towards the same as 10000, 943, 529, and 435 respectively; and therefore their densities are as 100, $94 \frac{1}{2}, 67$, and 400" [Newton 1999, p. 398].

7 “.... and the squares of their periodic times are as 29 to 5 , and their densities as 400 to $94 \frac{1}{2} \ldots$ Therefore the diameter of Jupiter from east to west is to its diameter from pole to pole nearly as $10 \frac{1}{3}$ to $9 \frac{1}{3}$ ", [Newton 1999, p. 408]. 
more general question of how the Earth deforms, if it is subjected not only to its own gravitational field and to centrifugal accelerations, but also to the gravitational influence of external celestial bodies, such as the Moon and the Sun. Traditionally we refer to "tides" when we think of the rise and fall of sea levels, dictated by the Moon's or the Sun's periodic gravitational pull. However, for geologists and astrophysicists the meaning of this term is much wider. For them, quite general, any permanent or periodic movement of the surface of the Earth, water or land, resulting from internal or external forces are tides. In what follows we will concentrate on the competing effects of self-gravity and centrifugal accelerations only. The influence of external bodies will be ignored.

Today we would say that if we wish to model the deformation of the Earth when subjected to forces we need an appropriate constitutive model. In particular, if we want to emphasize Earth's solid characteristics we need constitutive models pertinent to solids. Clearly, the concept of constitutive equations was in its infancy when this need arose first and, hence, Sommerfeld explains to us in great detail [Klein and Sommerfeld 2012, p. 685] that the transition between a fluid and a solid can be gradual. In fact, the only constitutive law available in the middle of the nineteenth century that had a sound mathematical basis and could therefore be used to study three-dimensional continua was Hooke's law of linear elasticity. Moreover, it is fair to say that tensor notation was still under development at that time and in the very few papers dedicated to the problem of a sphere subjected to general gravitation, i.e., tides, plus centrifugal forces, a most repelling notation is used. Further below we will revisit the problem in modern form. Thus, at this point it may suffice to mention that, at least to our knowledge, the first source that presents an explicit formula for the flattening of a rotating, self-gravitating, compressible, linear-elastic sphere is Thomson and Tait's treatise [1912, p. 432], which in modern notation reads

$$
f=\frac{\rho_{0} R^{2} \omega_{0}^{2}}{E} \frac{(1+v)(2+v)}{7+5 v} .
$$

$E$ denotes Young's modulus, $v$ is Poisson's ratio, and $\rho_{0}$ and $R$ are the mass density and the radius of the sphere in its reference configuration, respectively. Of course, the question arises which elastic constants to use for the Earth. If we take the ones for steel, as suggested in [Klein and Sommerfeld 2012, p. 692], i.e., $E_{E} \approx 210 \mathrm{GPa}$, and $v_{E} \approx 0.3$, we find with $\rho_{0, E} \approx 5514 \frac{\mathrm{kg}}{\mathrm{m}^{3}}$ and $R_{E}=6.371 \cdot 10^{6} \mathrm{~m}$, the mean radius of the Earth [Wikipedia 2016a; 2016c], a value of $f_{E}=2 \cdot 10^{-3}$, and we may conclude that a solid Earth model leads to an underestimate of the observed figure; in short, this Earth model is too rigid.

In fact, the question of which radius and which Young's modulus to use is rather subtle and it takes some care to find good answers. Let us start with the radius. The 
effect of self-gravitation of Earth or any other massive celestial object on deformation is immense. An originally homogeneous sphere of a mass equivalent to Earth will shrink considerably after "gravitation has been switched on." It was shown [Müller and Weiss 2016, p. 72] that the radius of the undeformed homogeneous sphere would shrink by more than ten percent, a number that calls for the use of geometrically nonlinear deformation theory. However, the radius of the initially homogeneous, unstrained sphere is not the radius $R_{E}$ that was inserted above in (1-4). Why? For an answer first note that the strength of centrifugal acceleration is two orders of magnitude below that of self-gravity. It is therefore justified to use linear elasticity (i.e., a linear relationship between stress and stain) and a linear strain measure in order to assess its effects on deformation. However, linear elasticity does not distinguish between the current and the reference configuration. The deformation is assumed to be small. In fact, exactly this kind of approximation was the foundation of (1-4). To say it once more, in problems of linear elasticity the size of the reference and of the current radius hardly differ. Hence, we may consider the quantity $R$ in (1-4) to be that of the deformed sphere subjected to self-gravity. Centrifugal accelerations will not change this value considerably. If one so wishes, we may alternatively say that the Earth deformed by self-gravity was considered as a new reference configuration onto which centrifugal loads were applied.

Now let us turn to the question of which Young's modulus is to be used in (1-4). Due to the authority of Lord Kelvin's and Love's statements according to which the resilience of Earth is basically that of steel, it is customary to use the corresponding elastic data: "Hence it appears that if the rigidity of the earth, on the whole, were only as much as that of steel or iron, the earth as a whole would yield about two-fifths as much to the tide-producing influences of the sun and moon as it would if it had no rigidity at all; and it would yield by more than three-fourths of the fluid yielding, if its rigidity were no more than that of glass" [Thomson 1863, p. 574], "The comparison between theory and observation, owing to the extreme complexity of the circumstances, has been hitherto so imperfect that we cannot say it disproves this result; and therefore, from tidal phenomena hitherto observed, we cannot infer that the earth is more effectively rigid than steel" [Thomson 1863, p. 576], and "An example will make it clear how the table is to be interpreted. From the first column we learn that a homogeneous sphere of the size and mass of the earth made of a material nearly as rigid as steel and nearly as incompressible, the Poisson's ratio of the material being $\frac{1}{4}$, could not exist. It would be unstable as regards radial displacement. If such a sphere existed for an instant it would at once begin to condense towards the centre. If it were as rigid and compressible as steel, and homogeneous, it would be stable" [Love 1911, p. 114].

However, Earth contains a nickel-iron core surrounded by a silicate mantle. In other words, Earth is a composite consisting of spherical shells. In such a situation 
it is more appropriate to use effective elastic properties instead, which could be obtained by a homogenization scheme. To the best knowledge of the author this has not been attempted so far. ${ }^{8}$ The simplest homogenization schemes would be to use Voigt and Reuss upper and lower volumetric bounds, i.e.,

$$
E_{\mathrm{Voigt}}=E_{\mathrm{Fe}} v+E_{\mathrm{SiO}_{2}}(1-v), \quad E_{\mathrm{Reuss}}=\left(\frac{v}{E_{\mathrm{Fe}}}+\frac{1-v}{E_{\mathrm{SiO}_{2}}}\right)^{-1}, \quad v=\left(\frac{r_{i}}{r_{o}}\right)^{3} .
$$

If we now take values for the elastic moduli of iron and silicon, $E_{\mathrm{SiO}_{2}}=80 \mathrm{GPa}$, and make use of the data for the radii of the iron core and the silicate mantle, $r_{i}=5100 \mathrm{~km}$ and $r_{o}=6370 \mathrm{~km}$, respectively, we find that the homogenized Young's modulus will be between $56 \%$ and $70 \%$ of the value for steel. After inserting this into (1-4) the value for the flatting will increase and come a little closer to the experimentally observed value.

Summarizing we may conclude that the Earth is a complex material body made of fluidic and solid-like shells. Indeed, it is likely that the fraction of both has not been constant in time, and that the current shape of the Earth may have been reached when the fraction was considerably different from today. Note that it is commonly assumed that the Earth was still almost completely in a fluid state $3 \mathrm{Ga}$ ago and then started to continuously form more and more outer solid crust [Turcotte and Pflugrath 1984]. Moreover, it is known today [Dehant et al. 2003] that as the Earth cools, the inner core solidifies from the fluid outer core. It can be assumed that such dynamic processes, even though they may be slow, will also lead to a change in the flattening. However, the description of such complex processes is far beyond the scope of this paper.

Compressible fluid or gas models. We shall finally consider the history of modeling the flattening of gas spheres; in other words we shall consider revolving stars. The question is what we mean by "gas". Surely a normal star like our Sun consists mostly of hydrogen and helium. From a very naive point of view, these are "gases". However, both are in an extreme state, temperature- and pressurewise, which is known as plasma. Thus, besides mechanical equilibrium it is imperative to study thermal equilibrium as well, in combination with quantum mechanics and, if the star is very heavy, as in the case of white dwarfs and neutron stars, by using relativistic instead of Newtonian gravity models. It is for that reason that scientific analysis of the deformation in rotating stars is comparatively young. As a matter of fact, in such cases the line between what we may want to call a fluid or a gas becomes indistinct. As we shall see, everything centers around the question of which equation of state to use in order to connect pressure, density, and temperature.

${ }^{8}$ A private communication by Igor Sevostianov from New Mexico State University indicates that NASA is in the process of doing that. 
In other words the stress tensor is isotropic and the capability for carrying shear forces is (mostly) ignored in such compressible fluid-gas models.

The story starts with two seminal papers, one by Milne [1923] and the other one by Chandrasekhar [1933]. Just like Newton they assume static equilibrium of forces to hold, such that the state of stress is spherically isotropic, given by a pressure, $p$. This pressure is connected to density $\rho$ via a so-called polytropic equation of state

$$
p=K \rho^{(n+1) / n},
$$

where $n$ is the polytropic index, a fudge parameter, and $K$ is a constant, which can be linked, for example, to the conditions at the center of the gas sphere. Moreover, the gas is considered as ideal; i.e., $p=\rho(R / M) T$ holds, where $R=8.314 \frac{\mathrm{kJ}}{\mathrm{kg} \cdot \mathrm{K}} \mathrm{de}-$ notes the ideal gas constant, $M$ the molecular weight, and $T$ absolute temperature.

Clearly, these relations account for compressibility, as it should be in the case of a gas. However, from the materials theory point of view we must ask what the status of the polytropic equation is and whether it qualifies as a true constitutive relation. The proper answer is that it does not. Rather it is a half breed. This is easily seen if one specializes (1-6) to the adiabatic equation of an ideal gas, for which $n=\frac{3}{2}, \frac{5}{2}$, or 3 depending on whether the ideal gas is monatomic, diatomic, or multiatomic. As is well known the adiabatic equation results from combination of the ideal gas law, the internal energy of an ideal gas, and the assumption to consider nonviscous, isentropic processes. In other words, it requires the first and second laws of $p \mathrm{~d} V$ thermodynamics to hold in combination with the two aforementioned constitutive equations for the ideal gas.

Interestingly, if we accept all this, a relation similar to (1-2) results in

$$
f=\frac{5}{4} \frac{\omega_{0}^{2}}{G \rho_{m}} v(n),
$$

where $\rho_{m}$ is the average of the inhomogeneous mass density distribution, and $v(n)$ is a function depending on the polytropic index, which must be determined numerically from a solution of a modified Lane-Emden equation. ${ }^{9}$ More details of the derivation and further references can be found in [Rozelot et al. 2011; Horedt 2004].

It should be mentioned that the flattening of our Sun is extremely small. While the difference of the polar and equatorial diameters of Earth is of the order of forty kilometers, it is merely $12 \mathrm{~km}$ in the case of the Sun [Rozelot et al. 2011, p. 175], despite the fact that the Sun is so much larger than Earth. In view of the much stronger gravitational pull we may conclude that the Sun "holds on very tightly"

\footnotetext{
${ }^{9}$ The modification is due to the fact that the original Lane-Emden equation was derived for a nonrotating star. Now the centrifugal accelerations had to be added.
} 
to its revolving masses. However, on second glance the situation is much more complicated. While the models described so far may be realistic when predicting the flattening of Earth, which seems essentially to rotate like a rigid body at a single angular speed, this is not so in the case of the Sun.

In this context astrophysicists have coined the term "differential rotation", which is supposed to mean that different parts of a rotating object move with different angular velocities at different latitudes and/or depths of the body and/or in time. All of this is happening in the Sun: Galileo's paper on sunspots [Galilei 1613] is often hailed to be the first definite scientific work on them including the observation that they are located on or close to the Sun's surface and that they move, because the Sun rotates. Galileo says on p. 27, “... il [a planet] quale oltre alla velocità douerebbe ancore muouersi quasi uniformemente... il che non accade nel moto delle macchie, le quali velocemente trepassano le parti di mezzo [of the Sun], e quanto più sono vicine alla circonferenza, tanto più pigramente caminano."10 However, with certainty we may say that this phenomenon was known a long time before, albeit not truly quantified, and that there is a long debate about priority rights, in which even the ever-so-modest Galileo did not hesitate to engage. We leave this uncommented here and refer to [Van Helden 1995; Galilei and Scheiner 2010] if the reader wishes to know more about this delicate topic.

Probably the first to make a quantitative statement about different angular speed in various latitudes of the Sun was Carrington [1859]. He says quite clearly on pp. 83-84 of his paper that "...there is an equatoreal current causing spots to move in the direction of the solar rotation, and a reverse current in the higher latitudes north and south..." and he quantifies this by daily drift recordings in form of a table on p. 83 of his paper. The reason for differential rotation in the Sun is due to strong mass convection, because of the steep temperature gradients from the core outwards. The convecting mass carries a portion of the star's angular momentum, so that the angular velocity must redistribute according to the principle of conservation of angular momentum.

However, incorporating differential rotation in models for the flattening did not happen until the 1980s, more than fifty years after the extension of the LaneEmden equation to a uniformly rotating star in [Milne 1923; Chandrasekhar 1933]. An overview on existing models is given in [Rozelot et al. 2011, p. 170; Horedt 2004 , §3.5]. Clearly, the numerical analysis is now even more complex. It is not surprising that no rule of thumb formulae for the flattening are presented any more. However, there are plots predicting the flattening according to various differential

10،... It [a planet], besides its velocity, would also have to move almost uniformly, being some considerable space away from the Sun... This does not happen with the motion of the sunspots, which traverse the middle region [of the Sun] with great rapidity, while the closer they are to the circumference, the more sluggishly they proceed" [Galilei and Scheiner 2010, p. 104]. 

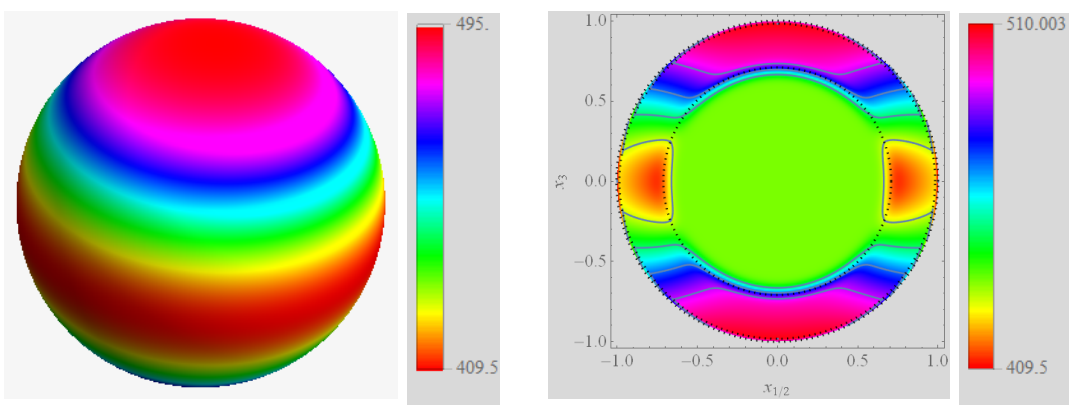

Figure 3. Distribution of the Sun's angular velocity field. Left: surface plot. Right: meridional section.

rotation models and these plots are commented verbally. One example of such work is [Godier and Rozelot 2000]. There an empirical equation for the angular velocity field $\omega(x, \theta)$ by [Kosovichev 1998; Godier and Rozelot 1999; 2000] is used, where $x$ is the normalized radial distance and $\theta$ is the polar angle:

$$
\begin{aligned}
\omega & =2 \pi\left(A_{1}(x)+A_{3}(x)[1-5 \cos \theta]+A_{5}(x)\left[1-14 \cos ^{2} \theta+21 \cos ^{4} \theta\right]\right), \\
A_{1}(x) & = \begin{cases}435, & x \leq 0.71, \\
435+51.85(x-0.71), & 0.71<x \leq 0.983, \\
435-882.53(x-1), & 0.983<x \leq 1,\end{cases} \\
A_{3}(x) & =-22 \Phi(x), \quad A_{5}(x)=-3.5 \Phi(x), \quad \Phi(x)=0.5\left(1+\operatorname{erf}\left[2 \frac{x-0.69}{0.1}\right]\right) .
\end{aligned}
$$

To be precise, it is assumed that the Sun rotates according to $\omega=\omega \boldsymbol{e}_{3}$, viewed from an inertial observer situated at the Sun's center with its unit vector $\boldsymbol{e}_{3}$ pointing to the Sun's pole. Figure 3 gives an idea of the complexity of the field. It is interesting to note that the core rotates quite differently than the rest of the Sun. As far as the flattening is concerned the conclusion is that "Comparing the profile of $\epsilon$ [the flattening] for the differential rotation case with the profile of $\epsilon$ in the rigid rotation case, where we find $\epsilon_{0} \simeq 2.59 \cdot 10^{-6}$, we see that the oblateness of the Sun is increased by the differential rotation of a quantity equal to $6.18 \cdot 10^{-6} \ldots$ " [Godier and Rozelot 2000, p. 370]. Still being a very small value the refined theory nevertheless makes a remarkable difference.

Most recently, the deformation of more exotic rotating stars has gained the attention of the astrophysicists, namely white dwarfs and neutron stars. For those readers who are not familiar with the nomenclature, we attempt to summarize facts in a somewhat dilettante manner. A white dwarf is a stellar remnant composed mostly of electron-degenerate matter. It is very dense. While its mass is comparable to that of the Sun, its volume is close to that of Earth. White dwarfs are thought to be the final evolutionary state of stars whose mass is not high enough to become a 
neutron star. The latter results from the gravitational collapse of the core of a star with a mass more than ten times that of the Sun. Neutron stars are composed almost entirely of neutrons. The conservation of angular momentum requires that neutron stars rotate extremely fast. A newborn neutron star can rotate many hundred times a second, so they say, which leads to the emission of electromagnetic beams that can be detected on Earth. For this reason neutron stars are also known as pulsars (= pulsating stars). Clearly, such a high spinning rate should lead to considerable deformation of the neutron star, which might result in a "starquake" during which its crusts "breaks". Indeed, changes in the pulsating emission pattern are observed, also known as "glitches", which are interpreted by the astrophysicists to be the result of exactly such an occurrence [Franco et al. 2000].

We now proceed to present Newton's fluid and the Hookean solid model of Thomson and Tait for revolving celestial objects in the language of modern continuum mechanics.

\section{Fluid models}

The following sections were adapted in part from [Müller and Lofink 2014]. Some further information regarding the derivation of pertinent formulae can be found in Appendix A. The issue of instabilities during revolution of fluidic, self-gravitating spheres has been addressed in a seminal paper by Poincaré [1885]. However, the angular speed of the Earth and other bodies of our solar system are small enough to ignore such peculiarities in this paper.

Discussion of pressure and flattening relations. We consider a self-gravitating sphere made of an incompressible fluid which starts spinning about a fixed axis at a constant angular velocity, $\omega_{0}$. Due to centrifugal accelerations and internal friction it finally reaches a stationary state of axisymmetric deformation and assumes the shape of a spheroid with major and minor axes $a$ and $c$, respectively. Its flattening, $f=1-\lambda$, can be calculated from the transcendental equation

$$
\frac{2 \omega_{0}^{2} a^{3}}{3 G m}=\frac{\left(1+2 \lambda^{2}\right) \arccos \lambda-3 \lambda \sqrt{1-\lambda^{2}}}{\left(1-\lambda^{2}\right)^{3 / 2}}, \quad \lambda=\frac{c}{a} .
$$

An alternative but numerically equivalent solution in terms of $\arcsin \lambda$ was presented in [Fitzpatrick 2016, p. 46]. For a given angular velocity (2-1), allows one to calculate the flattening of a body of mass $m$ and equatorial radius $a$ numerically. For this purpose note that $f=1-\lambda$. Moreover, we can expand (A-14) in a series for the flattening:

$$
\frac{2 \omega_{0}^{2} a^{3}}{3 G m}=\frac{8}{15} f+\frac{44}{105} f^{2}+O[f]^{3}
$$


Obviously, the first term is dominant for small flattening values and agrees with Newton's result from (1-2). Interestingly MacLaurin was obviously the first who presented a solution to the flattening problem in terms of a series about $f \approx 0$ (which in hindsight explains the term MacLaurin series, i.e., a series close to the point zero). He says in Section 655 of [MacLaurin 1742, p. 543], "The gravity... represented... at the equator by $\mathrm{D}$, and the centrifugal force at $\mathrm{D}$ by $\mathrm{V}, \ldots$, if the density of the spheroid be uniform... the ratio of $\mathrm{V}$ to $\mathrm{D}$ may be determined to any degree of exactness, at pleasure.... The excess of the semidiameter of the equator above the semiaxis is to the mean semidiameter nearly as $5 \mathrm{~V}$ to $4 \mathrm{D}-\frac{11 \mathrm{~V}}{7}$." Now, (A-8) allows us to calculate the magnitude of gravitational acceleration at the equator of a spheroid:

$$
\mathrm{D}=\frac{3 G m}{2 a_{1}^{2}} \frac{1}{1-\lambda^{2}}\left[\frac{\arccos \lambda}{\sqrt{1-\lambda^{2}}}-\lambda\right] .
$$

And since the centrifugal acceleration at the equator of the spheroid is given by $\mathrm{V}=a \omega_{0}^{2}$, a series expansion leads to

$$
\frac{2 \omega_{0}^{2} a^{3}}{3 G m}=\frac{\frac{4}{5} f}{1+\frac{44}{35} f} \frac{1}{1-\lambda^{2}}\left[\frac{\arccos \lambda}{\sqrt{1-\lambda^{2}}}-\lambda\right]=\frac{8}{15} f-\frac{184}{525} f^{2}+O[f]^{3},
$$

if we accept the quoted result from MacLaurin's book. In comparison with (2-2) we conclude that the dominant (i.e., Newton's) term comes out correctly; the higherorder terms, however, do not. In fact, MacLaurin never claimed this to be the case, and it is fair to say that a closed-form solution, such as (2-1), does not appear in his treatise, as one could surmise from a remark on [Fitzpatrick 2016, p. 47].

The pressure distribution within the spheroid is given by (see Appendix A)

$$
\bar{p}\left(\bar{\xi}_{1}\right)=\bar{p}(\overline{\mathbf{0}})\left(1-\bar{\xi}_{1}^{2}\right), \quad \bar{p}(\overline{\mathbf{0}})=\frac{3 \bar{\rho}_{0} G m}{2 a}\left(1-\frac{\lambda}{\sqrt{1-\lambda^{2}}} \arccos \lambda\right) \frac{\lambda}{1-\lambda^{2}},
$$

where a comoving, so-called spherical radial coordinate [Sokolov 1992] has been used (see (A-16) for details). Note that for vanishing flattening, i.e., $\lambda=1$,

$$
\bar{p}\left(\bar{\xi}_{1}\right)=\frac{\bar{\rho}_{0} G m}{2 R}\left(1-\bar{\xi}_{1}^{2}\right) .
$$

In fact, the form of this equation corresponds to the result for the pressure distribution in a self-gravitating, nonrotating, homogeneous sphere of radius $a=c \equiv R$ : recall that the gravitational acceleration at a radial position $r$ within such a sphere is given by $\boldsymbol{f}=-G m(r) / r^{2} \boldsymbol{e}_{r}$ where $m(r)=\frac{4}{3} \pi \rho_{0} r^{3}$ is the total mass "beneath" that position [Müller 2014, p. 269]. Thus, from the analogue to (A-2) in an inertial frame we conclude that

$$
\nabla \cdot \boldsymbol{\sigma}=-\rho \boldsymbol{f} \quad \Longrightarrow \quad p(r)=-\frac{1}{2} \frac{4}{3} \pi G \rho_{0}^{2} r^{2}+C .
$$


The constant of integration is determined by the requirement of vanishing pressure at the outer surface of the sphere at the outermost radial position, $R$. Hence,

$$
p(r)=\frac{\rho_{0} G m}{2 R}\left(1-\frac{r^{2}}{R^{2}}\right), \quad m=\frac{4}{3} \pi \rho_{0} R^{3} .
$$

In comparison with (2-6) we may interpret the generalized spherical coordinate as a normalized measure of the radial distance from the center of the ellipsoid to an arbitrary point within. We may also be tempted to conclude that because (2-6) and (2-8) refer to pressure induced exclusively by gravitation, (2-5) includes only gravitational but no centrifugal effects. This seems even more plausible, because the angular velocity $\omega_{0}$ does not occur in this expression at all. This conclusion, however, is erroneous: alternatively, (A-12) allows us by using (A-15) $)_{2}$ to write for $\bar{p}(\overline{\mathbf{0}})$ in $(\mathrm{A}-17)$

$$
\bar{p}(\overline{\mathbf{0}})=\frac{3 \bar{\rho}_{0} G m}{4 a}\left(\frac{1}{\lambda \sqrt{1-\lambda^{2}}} \arccos \lambda-1\right) \frac{\lambda}{1-\lambda^{2}}-\frac{1}{2} \bar{\rho}_{0} \omega_{0}^{2} a^{2} .
$$

The first term is always positive and accounts for gravitational effects only. The second one is clearly negative and shows that the centrifugal acceleration leads to a pressure decrease. Moreover, if we insert (2-1) into (2-9), we reobtain (2-5). This proves that $\bar{p}(\overline{\mathbf{0}})$ of (2-5) is truly the net pressure including gravitational and centrifugal acceleration. Moreover, note that the pressure vanishes everywhere, provided $\lambda=0$, i.e., in the case of total flattening, $f=1$. Then according to (A-14) the corresponding, critical angular velocity is given by $\omega_{0}^{c}=\pi \sqrt{G \bar{\rho}_{0}}$. For a liquid Earth this would result in a revolution time of circa 3300 seconds. This is, of course, just a curious, totally unrealistic result.

Evaluation of the flattening formulae for the fluid model. The predictions for the flattening of various celestial bodies shown in Table 1 are based on experimental data presented in [Williams 2017; Rozelot and Neiner 2011, p. 179]. More specifically, the values shown in the column $f$ (Newton) were obtained by using Newton's result shown in (1-2). The numbers in the column $f$ (exact) are based on a numerical evaluation of (A-14).

Figures 4 and 5 allow us to compare the observed and the predicted values for the flattening of various planets and for some stars, respectively. The following can be said: intuitively we expect gas-like bodies, such as gas giants or "stars", to follow the fluid model quite closely. However, gaseous object or not, the fluid model tends to overestimate the flattening. In fact, Newton's formula leads to the highest overestimates. The numerical solution of (A-14) predicts amounts of flattening somewhat closer but (in general) still larger than the observed values. Note that in the case of Mars the predictions match reality surprisingly well. This is also true for Mercury and Venus. Indeed, no flattening has been observed for them. 


\begin{tabular}{|l|rccccl|}
\hline Planets & \multicolumn{1}{|c}{$\omega_{0}[1 / \mathrm{s}]$} & $a_{1}[\mathrm{~m}]$ & $m[\mathrm{~kg}]$ & $f$ (Newton) & $f$ (exact) & $f$ \\
\hline Mercury & $1.23993 \cdot 10^{-6}$ & $2.4395 \cdot 10^{6}$ & $3.300 \cdot 10^{23}$ & $1.26683 \cdot 10^{-6}$ & 0.0 & 0.000 \\
Venus & $-2.99242 \cdot 10^{-7}$ & $6.0520 \cdot 10^{6}$ & $4.870 \cdot 10^{24}$ & $7.63391 \cdot 10^{-8}$ & 0.0 & 0.000 \\
Earth & $7.30263 \cdot 10^{-5}$ & $6.3780 \cdot 10^{6}$ & $5.970 \cdot 10^{24}$ & $4.34083 \cdot 10^{-3}$ & $4.32631 \cdot 10^{-3}$ & $3.350 \cdot 10^{-3}$ \\
Mars & $7.09483 \cdot 10^{-5}$ & $3.3960 \cdot 10^{6}$ & $6.420 \cdot 10^{23}$ & $5.75157 \cdot 10^{-3}$ & $5.72576 \cdot 10^{-3}$ & $5.890 \cdot 10^{-3}$ \\
Jupiter & $1.76296 \cdot 10^{-4}$ & $7.1492 \cdot 10^{7}$ & $1.898 \cdot 10^{27}$ & $1.12071 \cdot 10^{-1}$ & $1.03131 \cdot 10^{-1}$ & $6.487 \cdot 10^{-2}$ \\
Saturn & $1.63115 \cdot 10^{-4}$ & $6.0268 \cdot 10^{7}$ & $5.680 \cdot 10^{26}$ & $1.92058 \cdot 10^{-1}$ & $1.67431 \cdot 10^{-1}$ & $9.796 \cdot 10^{-2}$ \\
Uranus & $-1.01473 \cdot 10^{-4}$ & $2.5559 \cdot 10^{7}$ & $8.680 \cdot 10^{25}$ & $3.70974 \cdot 10^{-2}$ & $3.60525 \cdot 10^{-2}$ & $2.293 \cdot 10^{-2}$ \\
Neptune & $1.08406 \cdot 10^{-4}$ & $2.4764 \cdot 10^{7}$ & $1.020 \cdot 10^{26}$ & $3.27717 \cdot 10^{-2}$ & $3.19531 \cdot 10^{-2}$ & $1.708 \cdot 10^{-2}$ \\
Pluto & $-1.13851 \cdot 10^{-5}$ & $1.1950 \cdot 10^{6}$ & $1.310 \cdot 10^{22}$ & $3.16255 \cdot 10^{-4}$ & $3.16178 \cdot 10^{-4}$ & 0.000 \\
\hline Stars & & & & & & \\
\hline Sun & & & & & & \\
Achernar & $3.49445 \cdot 10^{-5}$ & $8.35610 \cdot 10^{9}$ & $1.3323 \cdot 10^{31}$ & 1.00160 & $5.83710 \cdot 10^{-1}$ & $3.103 \cdot 10^{-1}$ \\
Regulus & $1.09432 \cdot 10^{-4}$ & $2.89678 \cdot 10^{9}$ & $7.5565 \cdot 10^{30}$ & $7.21520 \cdot 10^{-1}$ & $4.72896 \cdot 10^{-1}$ & $2.453 \cdot 10^{-1}$ \\
Vega & $1.39475 \cdot 10^{-4}$ & $1.93583 \cdot 10^{9}$ & $4.2456 \cdot 10^{30}$ & $6.22579 \cdot 10^{-1}$ & $4.27247 \cdot 10^{-1}$ & $1.870 \cdot 10^{-1}$ \\
Alderamin & $1.44117 \cdot 10^{-4}$ & $1.96368 \cdot 10^{9}$ & $3.7782 \cdot 10^{30}$ & $7.79630 \cdot 10^{-1}$ & $4.97959 \cdot 10^{-1}$ & $2.296 \cdot 10^{-1}$ \\
Altair & $1.87638 \cdot 10^{-4}$ & $1.47625 \cdot 10^{9}$ & $3.5595 \cdot 10^{30}$ & $5.96023 \cdot 10^{-1}$ & $4.14304 \cdot 10^{-1}$ & $1.916 \cdot 10^{-1}$ \\
\hline
\end{tabular}

Table 1. Experimentally observed and predicted flattening values.

Data from last column from [Williams 2017; Rozelot and Neiner 2011].
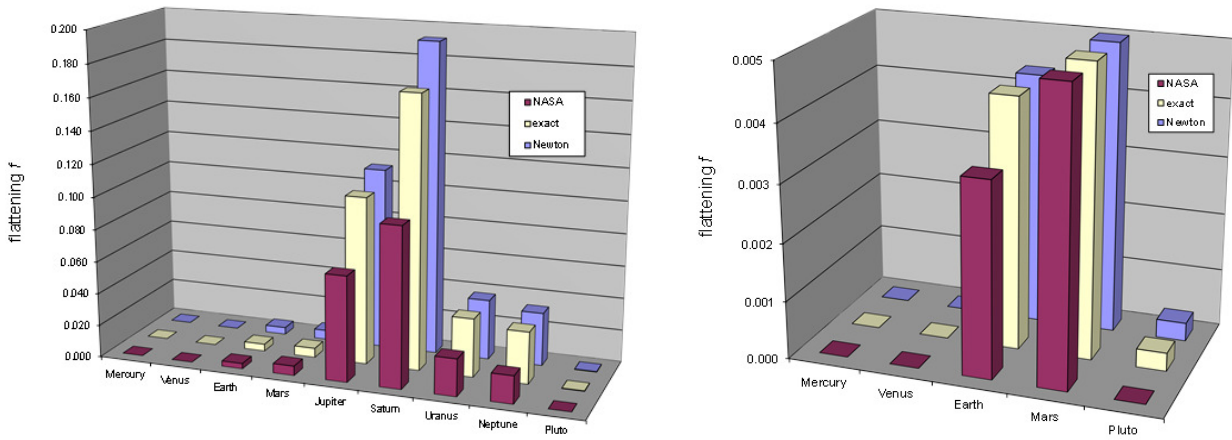

Figure 4. Comparison of observed and predicted flattening values for the planets.

One reason for the observed discrepancy is the assumption of incompressibility, i.e., a constant mass density $\bar{\rho}_{0}$ throughout the body. This is not a very realistic assumption, since the mass density increases, if we approach the center of the celestial body, where the heavy elements gather. They "sink" to the bottom, so to speak. The amount of densification has been quantified for the Earth in the PREM model [Dziewonski and Anderson 1981, p. 312], for gas giants such as Jupiter or Saturn in [Miles and Ramsey 1952], or for the Sun in [Blanch et al. 94, p. 43]. 


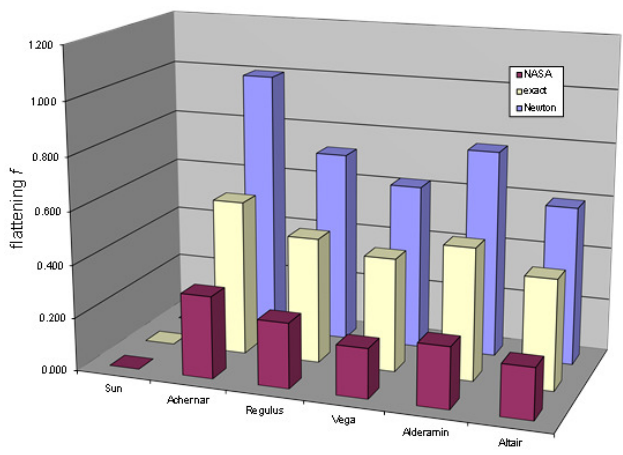

Figure 5. Comparison of observed and predicted flattening values for some stars.

Our simple fluid model does not allow for a radially varying density. However, the following simple argument shows in which direction the flattening will change if mass is accumulated closer to the center of an ellipsoidal fluid body rotating at a fixed angular speed. Suppose we compress the matter of an ellipsoid such that it occupies only half of the original equatorial radius. This will require its constant mass density to increase roughly by a factor of eight. According to (1-2) this would lead to a decrease of the flattening by one eighth. We may thus suspect that rearranging matter of a fixed amount toward the center will have the same effect.

The flattening of our Moon deserves a separate comment. The observed value of 0.0012 [Williams 2017] is small but distinct from zero. Of course we cannot simply apply (1-2) or (2-1) since the Moon "is tidally coupled to the Earth so that the same side of the Moon always faces the Earth, the rotation of the Moon is too small to explain the observed value of $J_{2}$ [the moment of inertia, $C$ ]. However, the present flattening may be a relic of a time when the Moon was rotating more rapidly. At that time the lunar lithosphere may have thickened enough so that the strength of the elastic lithosphere was sufficient to preserve the rotational flattening" [Turcotte and Schubert 2002, p. 377]. We may now use (2-1) and the data for mass and equatorial radius presented in [Williams 2017] to predict that the former rotation rate of the Moon was roughly 58 hours. Note that for the evaluation Figure 6 is very useful.

The other big moons of our solar system, like Io [Thomas et al. 1998], Europa [Van Hoolst et al. 2008], Ganymede [Anderson et al. 2001a], and Callisto [Anderson et al. 2001b] of Jupiter, or Titan of Saturn [Zebker et al. 2009] also show tidal coupling to their planet. According to the literature they seem to have no or almost no flattening, or, like Io a strongly ellipsoidal shape, which cannot be explained by our axisymmetric fluid model. The latter is also the case for large asteroids from the asteroid belt between Mars and Jupiter, which is why they are not studied here either. 

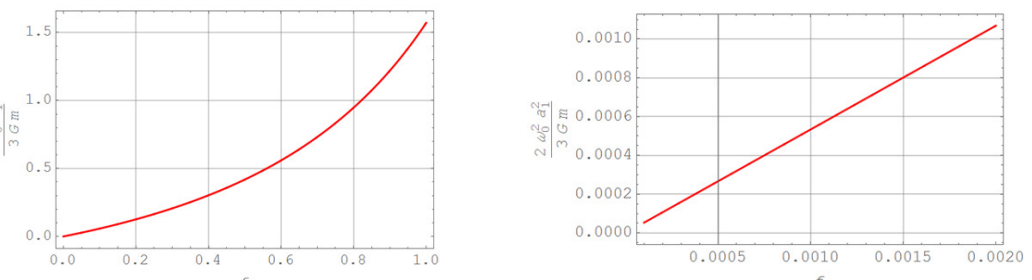

Figure 6. Graphic representation of (2-4) used for correlation of angular velocity and predicted flattening.
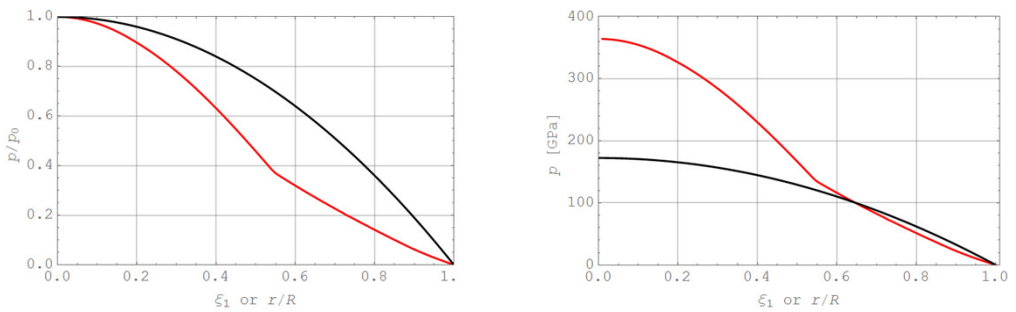

Figure 7. Modeling the pressure distribution within the Earth as a function of dimensionless radius, $\xi_{1}$, using an incompressible fluid model (black) and the PREM model (red).
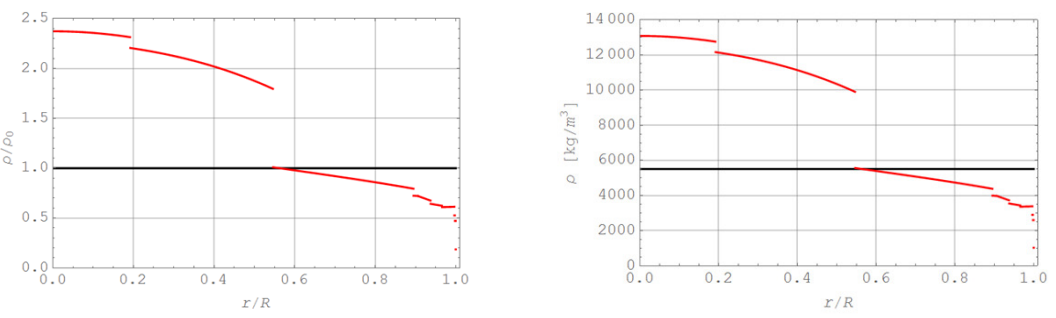

Figure 8. Mass density distribution within the Earth as a function of dimensionless radius, $r / R$, according to the PREM model (red) in comparison with the average mass density, $\rho_{0}=\rho(r=0)$.

We now discuss the evolution of the pressure as a function of quasiradial distance, $\bar{\xi}_{1}$, according to the incompressible fluid model. To this end we may start directly from (2-5), which is plotted in Figure 7 (black lines). The red lines stem from the so-called PREM model [Dziewonski and Anderson 1981], which is based on experimental evidence (elastic wave scattering) and allows for compressibility.

Obviously there is a distinct transition point (the end of the outer core), where the pressure shows a kink when plotted over the dimensionless radius, $r / R$. In this context, it is fair to point out that the PREM model considers the Earth as spherical. 
The reason for the transition is the huge jump in mass density when entering the outer core, i.e., essentially changing from the density of silicon dioxide to iron. The corresponding density plots are shown in Figure 8.

In Table I of the PREM model [Dziewonski and Anderson 1981] the mass density was fitted piecewise by splines, which is in agreement with the numerical data presented in the additional Table II. In the latter pressure data is also recorded. However, the pressure can be calculated as follows, once the density function $\rho=\rho(r / R)$ is known. We start from Poisson's equation for the gravitational potential in the inertial frame, specialized to the case of purely radial dependence:

$$
\begin{array}{r}
\Delta U^{f}=4 \pi \rho(\boldsymbol{x}) \Longrightarrow \frac{1}{r^{2}} \frac{\mathrm{d}}{\mathrm{d} r}\left(r^{2} \frac{\mathrm{d} U^{f}}{\mathrm{~d} r}\right)=4 \pi \rho(r) \Longrightarrow \frac{\mathrm{d} U^{f}}{\mathrm{~d} r}=\frac{m(r)}{r^{2}} \\
\text { with } m(r)=4 \pi \int_{r^{\prime}=0}^{r^{\prime}=r} \rho\left(r^{\prime}\right) r^{\prime 2} \mathrm{~d} r^{\prime} .
\end{array}
$$

This corresponds to the well known fact that the gravitational attraction in a sphere of purely radially dependent density is given by Newton's law for point masses as if the mass $m(r)$ "beneath" the radial point of interest were concentrated at $r=0$. We now turn to the local balance of momentum specialized to the case of a fluid at rest and to purely radial dependencies. Then this vector equation degenerates to

$$
\rho \frac{\mathrm{d} \boldsymbol{v}}{\mathrm{d} t}=\nabla \cdot \boldsymbol{\sigma}+\rho \boldsymbol{f} \quad \Longrightarrow \quad \frac{\mathrm{d} p}{\mathrm{~d} r}=-\rho(r) \frac{\mathrm{d} U^{f}}{\mathrm{~d} r} .
$$

After combining and integrating we obtain

$$
p(r)=p_{0}-\int_{r^{\prime}=0}^{r^{\prime}=r} \frac{\rho\left(r^{\prime}\right) m\left(r^{\prime}\right)}{r^{\prime 2}} \mathrm{~d} r^{\prime} .
$$

In fact, it is this equation that is depicted in Figure 7, which, of course, agrees with the pressure data presented in Table II of [Dziewonski and Anderson 1981]. Clearly, a radially dependent mass density is difficult to incorporate in the fluid model for the flattening presented above. However, in view of the extreme underestimate of the center pressure in the simple fluid model as evident in Figure 7 (right), it is absolutely essential to take it into account. In order to simulate the onion layered structure of the Earth we will discuss at the end of the paper the potential of combining the fluid and the solid model for predicting the flattening.

\section{Hookean solid model}

As in the case of the fluid model the following sections were repeated in part from [Müller and Lofink 2014]. However, typographical errors were removed and the way of presentation was changed for didactic reasons. 
Compilation of stresses and displacement formulae. It is shown in Appendix B that the stresses in a self-gravitating Hookean sphere are given by

$$
\begin{aligned}
\sigma_{r r}= & -\left[\frac{1}{10} \frac{3-v}{1-v}\left(\frac{g}{a_{c}}-\frac{2}{3}\right)+\frac{2}{3} \frac{3+2 v}{7+5 v} P_{2}\right]\left(1-\frac{r^{2}}{R^{2}}\right) \rho_{0} \omega_{0}^{2} R^{2}, \\
\sigma_{\vartheta \vartheta}= & -\left(\frac{1}{10} \frac{3-v}{1-v}\left(\frac{g}{a_{c}}-\frac{2}{3}\right)\left(1-\frac{1+3 v}{3-v} \frac{r^{2}}{R^{2}}\right)\right. \\
& \left.+\frac{3+2 v}{3(7+5 v)}\left[2 P_{2}\left(1-\frac{1}{3+2 v} \frac{r^{2}}{R^{2}}\right)+\frac{\mathrm{d}^{2} P_{2}}{\mathrm{~d} \vartheta^{2}}\left(1-\frac{2+v}{3+2 v} \frac{r^{2}}{R^{2}}\right)\right]\right) \rho_{0} \omega_{0}^{2} R^{2}, \\
\sigma_{\varphi \varphi}= & -\left(\frac{1}{10} \frac{3-v}{1-v}\left(\frac{g}{a_{c}}-\frac{2}{3}\right)\left(1-\frac{1+3 v}{3-v} \frac{r^{2}}{R^{2}}\right)\right. \\
& \left.+\frac{1}{3}\left[\left(\frac{2(1+v)}{4+5 v} P_{2}+\frac{2+v}{7+5 v}\right) \frac{r^{2}}{R^{2}}-\frac{3+2 v}{7+5 v}\right]\right) \rho_{0} \omega_{0}^{2} R^{2}, \\
\sigma_{r \vartheta}= & -\frac{3+2 v}{3(7+5 v)} \frac{\mathrm{d} P_{2}}{\mathrm{~d} \vartheta}\left(1-\frac{r^{2}}{R^{2}}\right) \rho_{0} \omega_{0}^{2} R^{2}, \\
\sigma_{r \varphi}= & 0, \quad \sigma_{\vartheta \varphi}=0 .
\end{aligned}
$$

Moreover, the displacements read

$$
\begin{aligned}
& u_{r}=-\frac{\rho_{0} \omega_{0}^{2} R^{2}}{E} \frac{(1+v)(1-2 v)}{1-v} {\left[\frac{1}{10}\left(\frac{g}{a_{c}}-\frac{2}{3}\right)\left(\frac{3-v}{1+v}-\frac{r^{2}}{R^{2}}\right)\right.} \\
&\left.\quad+\frac{2(1-v)}{3(1-2 v)} P_{2}\left(\frac{3+2 v}{7+5 v}-\frac{1+v}{7+5 v} \frac{r^{2}}{R^{2}}\right)\right] r, \\
& u_{\vartheta}=-\frac{\rho_{0} \omega_{0}^{2} R^{2}}{3 E}(1+v) \frac{\mathrm{d} P_{2}}{\mathrm{~d} \vartheta}\left(\frac{3+2 v}{7+5 v}-\frac{2+v}{7+5 v} \frac{r^{2}}{R^{2}}\right) r, \\
& u_{\varphi}=0 .
\end{aligned}
$$

For further investigations it will be advantageous to use Young's modulus, $E$, and Poisson's ratio, $v$, instead of Lamé's constants

$$
\lambda=\frac{v}{(1-2 v)(1+v)} E, \quad 2 \mu=\frac{1}{1+v} E .
$$

Moreover, we have defined the gravitational and centrifugal accelerations at the outer (equatorial) surface $r=R$ by

$$
g=\frac{G m}{R^{2}}, \quad a_{c}=R \omega_{0}^{2} .
$$

It will be instructive to divide the stresses and displacements into purely gravitational and centrifugal parts

$$
\sigma_{i j}=\sigma_{i j}^{\text {grav }}+\sigma_{i j}^{c}, \quad u_{i}=u_{i}^{\text {grav }}+u_{i}^{c}, \quad i, j \in\{r, \vartheta, \varphi\},
$$


where

$$
\begin{aligned}
& \sigma_{r r}^{\text {grav }}=-\frac{3 m g}{10 A} \frac{3-v}{1-v}\left(1-\frac{r^{2}}{R^{2}}\right), \\
& \sigma_{\vartheta \vartheta}^{\text {grav }} \equiv \sigma_{\varphi \varphi}^{\text {grav }}=-\frac{3 m g}{10 A} \frac{3-v}{1-v}\left(1-\frac{1+3 v}{3-v} \frac{r^{2}}{R^{2}}\right), \\
& \sigma_{r \vartheta}^{\text {grav }}=0, \quad \sigma_{r \varphi}^{\text {grav }}=0, \quad \sigma_{\vartheta \varphi}^{\text {grav }}=0, \\
& u_{r}^{\text {grav }}=-\frac{m g}{10 A k} \frac{1+v}{1-v}\left(\frac{3-v}{1+v}-\frac{r^{2}}{R^{2}}\right) r, \\
& u_{\varphi}^{\text {grav }}=0, \quad u_{\varphi}^{\text {grav }}=0,
\end{aligned}
$$

and

$$
\begin{aligned}
\sigma_{r r}^{c}= & \left(\frac{1}{10}-\frac{3+2 v}{7+5 v} P_{2}\right) \frac{2}{3}\left(1-\frac{r^{2}}{R^{2}}\right) \rho_{0} \omega_{0}^{2} R^{2}, \\
\sigma_{\vartheta \vartheta}^{c}= & \left(\frac{1}{15} \frac{3-v}{1-v}\left(1-\frac{1+3 v}{3-v} \frac{r^{2}}{R^{2}}\right)-\frac{3+3 v}{3(7+5 v)}\left[2 P_{2}\left(1-\frac{1}{3+2 v} \frac{r^{2}}{R^{2}}\right)\right.\right. \\
& \left.\left.\quad+\frac{\mathrm{d}^{2} P_{2}}{\mathrm{~d} \vartheta^{2}}\left(1-\frac{2+v}{3+2 v} \frac{r^{2}}{R^{2}}\right)\right]\right) \rho_{0} \omega_{0}^{2} R^{2}, \\
\sigma_{\varphi \varphi}^{c}= & \left(\frac{1}{15} \frac{3-v}{1-v}\left(1-\frac{1+3 v}{3-v} \frac{r^{2}}{R^{2}}\right)\right. \\
& \left.\quad-\frac{1}{3}\left[\left(\frac{2(1+v)}{7+5 v} P_{2}+\frac{2+v}{7+5 v}\right) \frac{r^{2}}{R^{2}}-\frac{3+2 v}{7+5 v}\right]\right) \rho_{0} \omega_{0}^{2} R^{2}, \\
\sigma_{r \vartheta}^{c} \equiv & \sigma_{r \vartheta}=-\frac{3+2 v}{3(7+5 v)} \frac{\mathrm{d} P_{2}}{\mathrm{~d} \vartheta}\left(1-\frac{r^{2}}{R^{2}}\right) \rho_{0} \omega_{0}^{2} R^{2}, \\
\sigma_{r \varphi}^{c}= & 0, \quad \sigma_{\vartheta \varphi}^{c}=0, \\
u_{r}^{c}= & \frac{\omega_{0}^{2} m}{2 \pi E} \frac{(1+v)(1-2 v)}{1-v}\left[\frac{1}{10}\left(\frac{3-v}{1+v}-\frac{r^{2}}{R^{2}}\right)\right. \\
& \left.\quad \frac{1-v}{1-2 v} P_{2}\left(\frac{3+2 v}{7+5 v}-\frac{1+v}{7+5 v} \frac{r^{2}}{R^{2}}\right)\right] \frac{r}{R}, \\
u_{\vartheta}^{c} \equiv & u_{\vartheta}=-\frac{\omega_{0}^{2} m}{2 \pi E}(1+v) \frac{\mathrm{d} P_{2}}{\mathrm{~d} \vartheta}\left(\frac{3+2 v}{7+5 v}-\frac{2+v}{7+5 v} \frac{r^{2}}{R^{2}}\right) \frac{r}{R}, \\
u_{\varphi}^{c}=0 . & 0 .
\end{aligned}
$$

$A$ denotes the surface area of the (spherical) celestial body. It is useful to introduce this quantity since the factor $\mathrm{gm} / \mathrm{A}$ can now be interpreted as the total "weight" of the celestial body distributed over its surface. This is nothing else but a pressure, and it serves nicely as a very intuitive measure for normalizing the gravitational stresses and displacement. However, of course, we may also write 

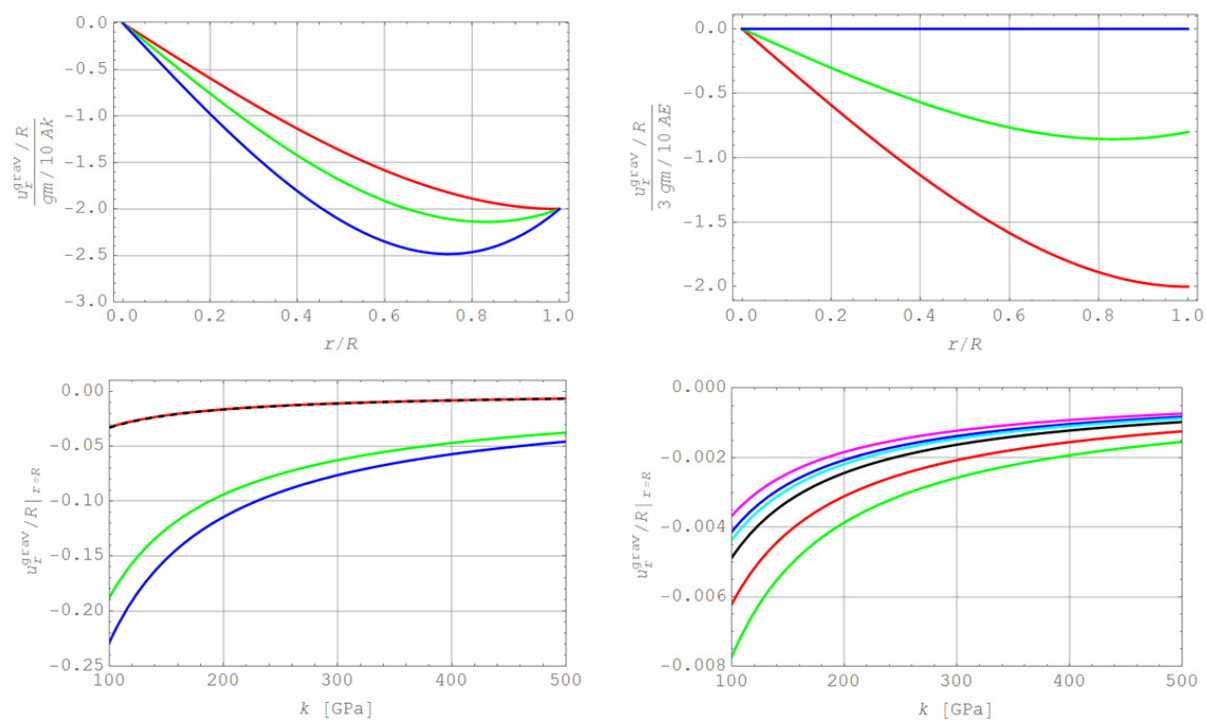

Figure 9. Behavior of the radial gravitational displacement component (see text).

$g m / A \equiv G m^{2} /\left(4 \pi R^{4}\right)$. As we shall see shortly this notation is more suitable if we wish to discuss the range of validity of the linear-elastic solution. Moreover, $k=\lambda+\frac{2}{3} \mu$ and $2 \mu=E /(1+v)$ denote the isotropic compressibility of a Hookean solid and its shear modulus, respectively. Gravity will compress the celestial body quite strongly and, therefore, it is most appropriate to use $k$ in context with the gravitational part of the solution.

Evaluation of the formulae for stresses and flattening. Figure 9 presents a study of various aspects of the behavior of the gravitational part of the displacement. In a sphere gravity leads to purely radial contraction, i.e., there is only a radial displacement, $u_{r}^{\text {grav }}$. The first two pictures concentrate on the dimensionless form given by (3-6) 6 . The situation is depicted for three different values of Poisson's ratio: $v=0$ (red), $v=0.3$ (green), and $v=0.5$ (blue). If we normalize by $E$ instead of $k$ (second picture in Figure 9), we can see very clearly that the radial contraction vanishes if $v=0.5$, i.e., if the body is incompressible, no matter how strong the gravitational force may be. This is an artifact inherent to the concept of incompressibility. It is interesting to note that, depending on Poisson's ratio, the extremum of $u_{r}^{\text {grav }}$ is not necessarily located at the surface of the celestial body. In fact, we find

$$
r^{\mathrm{ext}}=\sqrt{\frac{1}{3} \frac{3-v}{1+v} R}
$$




\begin{tabular}{|l|cll|}
\hline Moons & $a_{1}[\mathrm{~m}]$ & \multicolumn{1}{c|}{$m[\mathrm{~kg}]$} \\
\hline Moon & $1.738 \cdot 10^{6}$ & 7.34 & $\cdot 10^{22}$ \\
Io & $1.82 \cdot 10^{6}$ & 8.932 & $\cdot 10^{22}$ \\
Europa & $1.56 \cdot 10^{6}$ & 4.8 & $\cdot 10^{22}$ \\
Ganymede & $2.63 \cdot 10^{6}$ & 1.482 & $\cdot 10^{23}$ \\
Callisto & $2.41 \cdot 10^{6}$ & 1.08 & $\cdot 10^{23}$ \\
Titan & $2.576 \cdot 10^{6}$ & $1.3452 \cdot 10^{23}$ \\
\hline
\end{tabular}

Table 2. Physical data for the Moon and some Jupiter and Saturn moons [Williams 2017; Wikipedia 2015e; 2015d; 2015b; 2015c; 2015a; 2015f].

Note that at the surface of a celestial body we have

$$
u_{r}^{\text {grav }} /\left.R\right|_{r=R}=-\frac{G m^{2}}{20 \pi R^{4} k} .
$$

The concept of linear elasticity is valid if the displacements, and in particular this expression, remain small. This may not necessarily be so for all telluric celestial bodies, which we would like to treat as solids, in particular by the model of a Hookean solid. We proceed to investigate this issue in the next two plots.

Figure $9_{3}$ is dedicated to the inner planets (Mercury in red, Venus in green, Earth in blue, and the dashed line for Mars). For the numerical evaluation we have used the mass data shown in Table 1 . For the radius we use the values for $R \equiv a_{1}$. The latter choice is somewhat problematic: in order to meet the requirements of the linear theory of elasticity, we need to know the radius of the reference state, i.e., the outer radius before loads have been applied, and not a radius that includes the effects of gravity and centrifugal acceleration. Thus, our choice for $R$ represents essentially the current radial situation. However, within the framework of linear elasticity the difference between the current and the reference radius should differ by a few percent, at most. Moreover, the proper choice of $k$ is by no means obvious. Basically, $k$ is an average compressibility of the respective body. For that reason we have decided to depict (3-9) for a physically reasonable range of $k$ values. Clearly, the linear theory of elasticity seems to be applicable only to Mercury and Mars (for which the values nearly coincide). Venus and Earth show normalized displacements of $5 \%$ and more, which are not acceptable. A nonlinear approach is necessary to calculate the gravitational stresses and displacements in this case.

Figure 94 focuses on various moons (Earth's Moon in red, Io in green, Europa in blue, Ganymede in black, Callisto in magenta, and Titan in cyan). The necessary data is compiled in Table 2. We conclude that the linear-elastic solution applies.

The first two pictures in Figure 10 show the nonvanishing dimensionless components of the centrifugal part of the displacement according to (3-7) 7,8 for three 

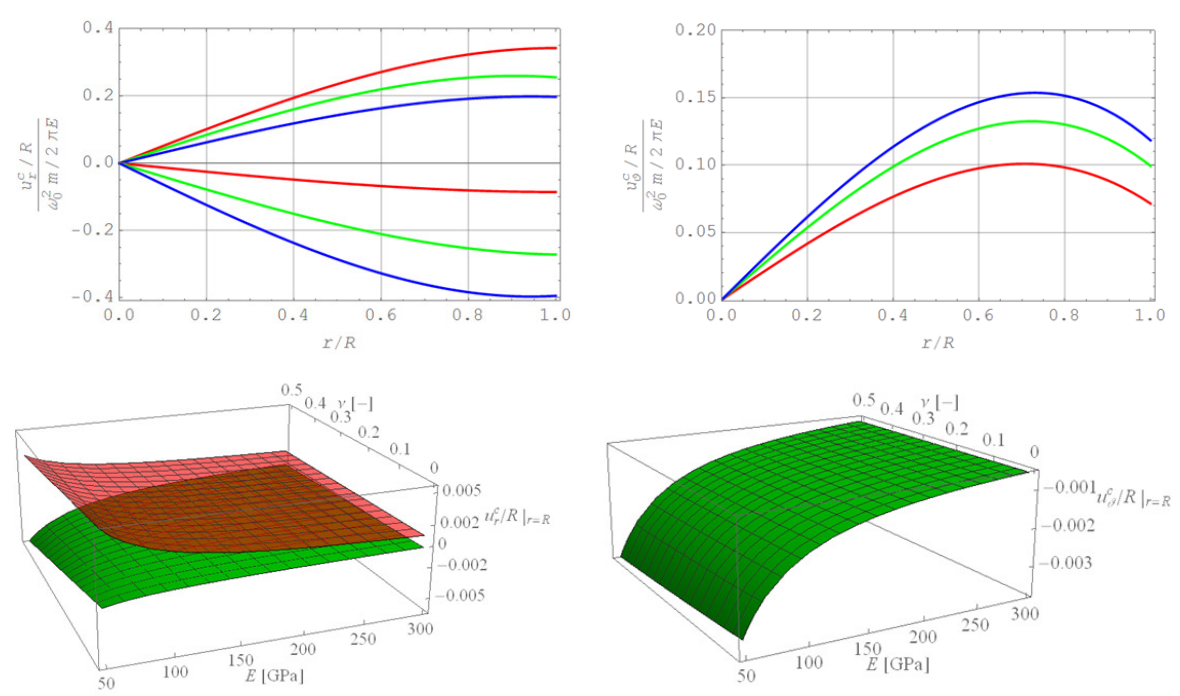

Figure 10. Behavior of the centrifugal displacement components (see text).

different values of Poisson's ratio, namely $v=0$ (red), $v=0.3$ (green), and $v=0.5$ (blue). Here $u_{r}^{c}$ was evaluated at the equator, i.e., $\vartheta=\pi / 2$, and for the pole, i.e., $\vartheta=0$. This leads to positive and to negative values, respectively, which makes sense in view of the effect of the centrifugal acceleration on a deformable body (extension perpendicular to the axis of rotation accompanied by lateral contraction). Further, $u_{\vartheta}^{c}$ was evaluated at the equator, i.e., $\vartheta=\pi / 4$, where it assumes its extremum. It is interesting to note that the extreme values are not necessarily located at the surface of the body and that the location depends on Poisson's ratio:

for $u_{r}^{c} \rightarrow r^{\mathrm{ext}}=\sqrt{\frac{1}{3} \frac{(1-2 v)(3-v) /(1+v)-10((1-v)(3+2 v) /(7+5 v)) P_{2}}{1-2 v-10((1-v)(1+v) /(7+5 v)) P_{2}}} R$,

for $u_{\vartheta}^{c} \rightarrow r^{\mathrm{ext}}=\sqrt{\frac{1}{3} \frac{3+2 v}{2+v} R} \quad$ (independently of $\vartheta$ ).

We now concentrate specifically on the Earth and find for the centrifugal displacement components on its surface:

$$
\begin{aligned}
& u_{r}^{c} /\left.R\right|_{r=R}=\frac{\omega_{0, E}^{2} m_{E}}{2 \pi E}\left[\frac{1}{5}(1-2 v)-\frac{(1+v)(2+v)}{7+5 v} P_{2}\right], \\
& u_{r}^{c} /\left.R\right|_{r=R}=\frac{\omega_{0, E}^{2} m_{E}}{4 \pi E}\left[\frac{1}{5}(1-2 v)-\frac{(1+v)(2+v)}{7+5 v} P_{2}\right] .
\end{aligned}
$$


The third and fourth pictures in Figure 10 illustrate these relationships when evaluated in the equatorial plane, $u_{r}^{c} /\left.R\right|_{\vartheta=\pi / 2}$ (positive values due to centrifugal acceleration), in the polar direction, $u_{r}^{c} /\left.R\right|_{\vartheta=0}$ (negative values due to lateral contraction, i.e., the Poisson effect), and at $45^{\circ}, u_{\vartheta}^{c} /\left.R\right|_{\vartheta=\pi / 4}$, using Earth data from Table 1 (with $R=a_{1}$ ) for physically reasonable ranges of Young's moduli and Poisson's ratios. Obviously, all values stay below the $1 \%$ threshold and, hence, the message is that linear elasticity may be used to describe the centrifugal displacements and stresses even in the case of the Earth. We now compute the flattening in general as

$$
\begin{aligned}
& a=R+ u_{r}(r=R, \vartheta=\pi / 2), \quad c=R+u_{r}(r=R, \vartheta=0) \\
& \quad \Longrightarrow \quad f \equiv \frac{a-c}{a} \approx \frac{\rho_{0} R^{2} \omega_{0}^{2}}{E} \frac{(1+v)(2+v)}{7+5 v} \equiv \frac{\rho_{0} R^{2} \omega_{0}^{2}}{\mu} \frac{1+v / 2}{7+5 v},
\end{aligned}
$$

if we neglect higher-order terms in $u_{r}$ as we should within the framework of a linear theory.

This, indeed, is the result originally presented by Thomson and Tait [1912, p. 432]. However, in comparison with (A-14) from the fluid model this relation has a serious drawback: for a given telluric body it is not evident which effective elastic constants, i.e., Young's modulus and Poisson's ratio, to use. However, if we believe that this simple Hookean model applies to telluric planets, we may use this result to determine an effective shear modulus or modulus of rigidity, $\mu \equiv G$, if we use the experimentally observed data for the flattening. The factor $(1+v / 2) /(7+5 v)$ is nearly constant for all possible values of $v$, i.e., circa $\frac{1}{7}$ :

$$
\mu=\frac{3 m \omega_{0}^{2}}{28 \pi R f} .
$$

If we evaluate this relation using Earth's data, we obtain a value of $50 \mathrm{GPa}$, which is smaller than the value for iron or steel (roughly $70 \mathrm{GPa}$ ), which is often quoted in context with planet Earth.

We now turn to the stresses and begin by examining the purely gravitational part shown in (3-6). As it should, these relations are of a purely radial nature: there are no shear stresses, all normal stresses depend only on the radius $r$, and the two angular stresses are equal. Figure 11 illustrates their dependence on $r$ for three different choices of Poisson's ratio: $v=0$ (red), $v=0.3$ (green), and $v=0.5$ (blue). The maximum compression is at the body's center. Interesting to note is the cross-over point of the angular stresses. It is independent of Poisson's ratio and located at $r / R=\sqrt{1 / 2}$.

It should be pointed out that the linear-elastic solution for the gravitational part is dominant in comparison with the stresses due to centrifugal accelerations. Figure 12 illustrates the situation by showing the behavior of all combined stresses 

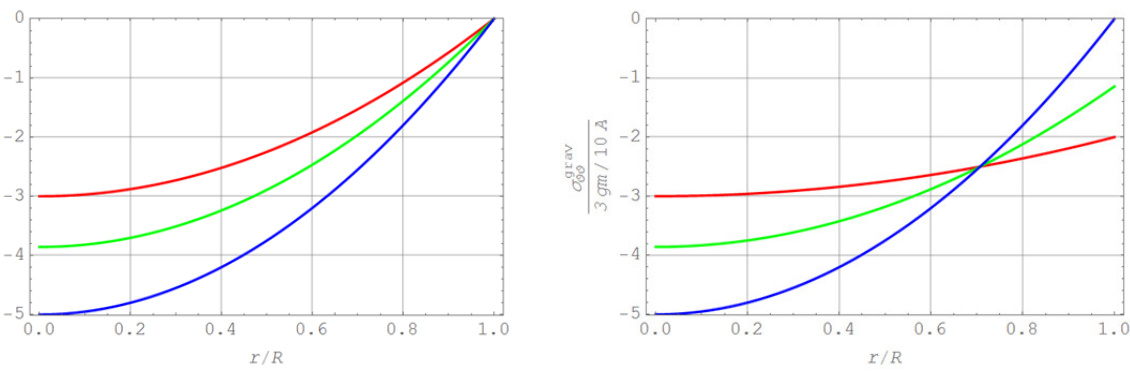

Figure 11. Behavior of the gravitational stress components (see text).
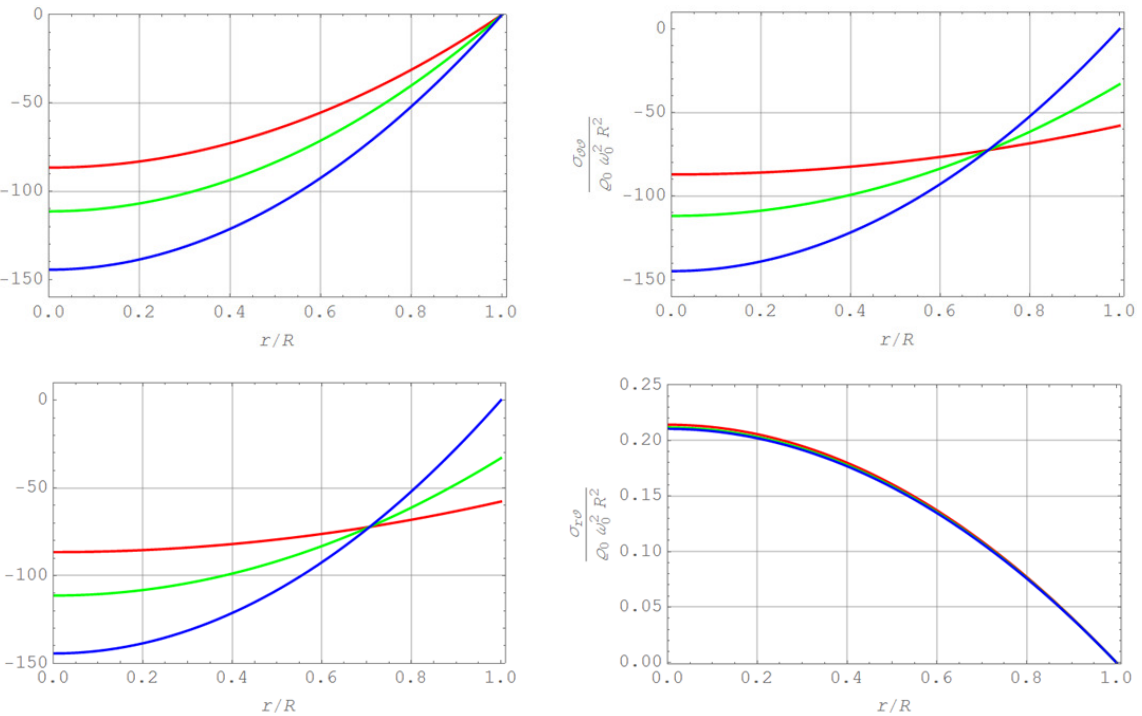

Figure 12. Behavior of the combined stress components (see text).

according to (3-1) as a function of radial position for various values of Poisson's ratio. In fact, the plots for the normal stresses were generated for the equatorial plane, i.e., by choosing $\vartheta=\pi / 2$, and the one for the shear stress at $\vartheta=\pi / 4$ in order to show the maximum values. For the numerical evaluation we have chosen the observed mean radius of the Earth, i.e., $R_{E}=6.371 \cdot 10^{6} \mathrm{~m}, \omega_{0, E}=2 \pi / 86164 \mathrm{~s}$, and $m_{E}=5.97 \cdot 10^{24} \mathrm{~kg}$ [Wikipedia 2016a; 2016c]. Thus, we have $g=9.81 \frac{\mathrm{m}}{\mathrm{s}^{2}}$, $a_{c}=0.034 \frac{\mathrm{m}}{\mathrm{s}^{2}}$, and $g / a_{c}=298.7$. These numbers already indicate the dominance of gravitation. In fact, in the case of the normal stresses it turns out that the gravitational parts in (3-1) are so strong that they conceal the dependence on the polar angle almost completely. All normal stresses are highly compressive. Note the striking similarity to the plots shown in Figure 11 and the very slight difference 

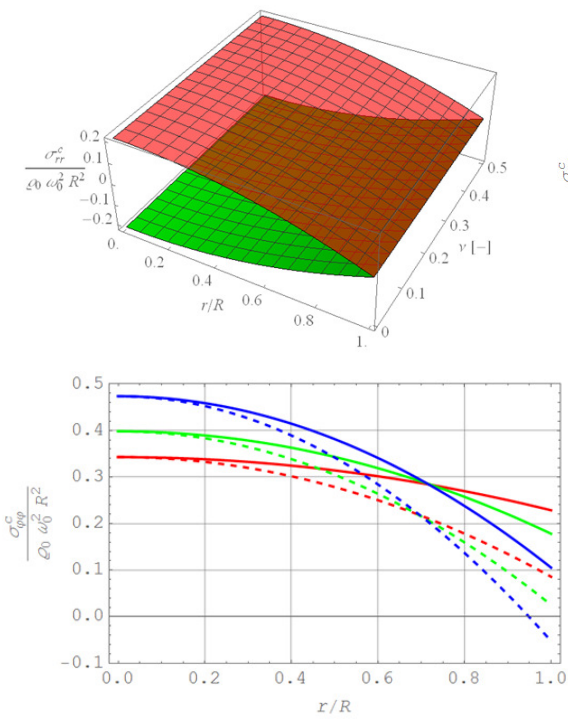
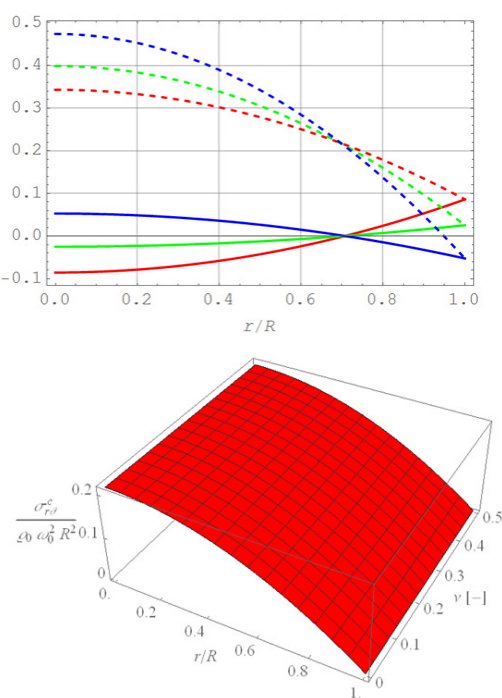

Figure 13. Behavior of the centrifugal stress components (see text).

between the two angular stresses. Both emphasize our point that gravitation is dominant. Moreover, the shear stress is hardly dependent on Poisson's ratio.

There is another caveat we have to keep in mind, specifically in context with the Earth. During our discussion of the displacements due to gravitation we found that the linear-elastic solution is not really valid for planet Earth: the predicted displacements were simply too large (see Figure $9_{3}$ ). To be specific, the radius we chose for our numerical evaluation of the stresses in Figure 12 is the observed mean radius, i.e., the radius after gravitation and centrifugal accelerations are "switched on". The symbol $R$ in our linear-elastic calculations, however, is the radius of the unloaded configuration. In other words, it is much larger than the chosen $R_{E}=$ $6.371 \cdot 10^{6} \mathrm{~m}$. Thus, the predicted magnitude of the combined normal stresses is doubtful, too: our numerical value underestimates distances in the reference configuration and the ratio $g / a_{c} \equiv G m_{E} /\left(R_{E}^{3} \omega_{0, E}^{2}\right)$ will become smaller. Most likely it will keep its dominance in the stress expressions, but the details are left to a nonlinear analysis and future research.

There is no problem with a numerical evaluation of the shear stress, though, since it is purely due to centrifugal acceleration. For conversion of the numbers shown on all plots in Figure 12 into absolute stress values we may use $\rho_{0, E} R_{E}^{0} \approx$ $119 \mathrm{GPa}$ in the case of the Earth. Thus, the shear stresses within this simplistic model are very small. For example, they amount to circa $0.1 \mathrm{MPa}$ one kilometer below the Earth's surface. This is in favor of the low shear stress hypothesis as 

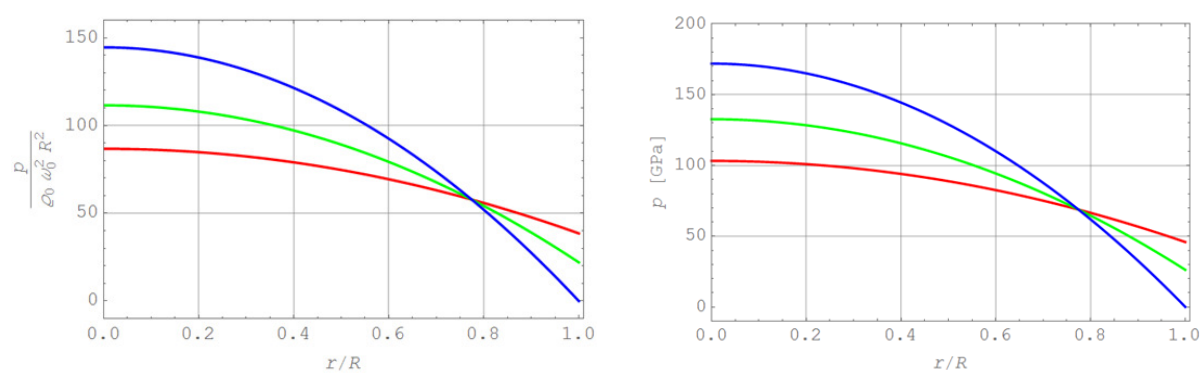

Figure 14. Mechanical pressure as a function of the dimensionless radius, $r / R$ (see text).

outlined, e.g., on [Lee et al. 2002, p. 543]. However, we have to keep in mind that this is a very simplistic Earth model, although an exact and quantitative one.

Figure 13 illustrates the behavior of all stress components due to centrifugal acceleration as given by (3-7) $1-6$. The radial as well as the shear stress show hardly any dependence on Poisson's ratio. Their behavior is depicted in Figure 13,4. There $\sigma_{r r}^{c}$ was evaluated along the equator at $\vartheta=\pi / 2$ and along the radius leading to the pole, i.e., $\vartheta=0$, giving positive and negative values, respectively, as intuitively expected. Further, $\sigma_{r \vartheta}^{c}$ was drawn for $\vartheta=\pi / 4$ at the location of maximum values. The angular normal stresses show a distinct dependence on $v$. They were evaluated for three different choices of Poisson's ratio: $v=0$ (red), $v=0.3$ (green), and $v=0.5$ (blue) at $\vartheta=\pi / 2$ (solid lines) and $\vartheta=0$ (dashed lines).

We now turn to a study of the mechanical pressure. If we restrict ourselves to gravitation we may write

$$
p^{\text {grav }}=-\frac{1}{3}\left(\sigma_{r r}^{\text {grav }}+\sigma_{\vartheta \vartheta}^{\text {grav }}+\sigma_{\varphi \varphi}^{\text {grav }}\right)=\frac{g m}{10 A} \frac{3-v}{1-v}\left(3-\frac{5(1+v)}{3-v} \frac{r^{2}}{R^{2}}\right) .
$$

Note that because of $3 \mathrm{gm} / A \equiv \rho_{0} \mathrm{Gm} / R$ this reduces to the pressure distribution for the gravitationally stressed, incompressible liquid sphere shown in (2-8), if we only use the incompressibility condition $v=0.5$ for a Hookean solid. We might have suspected this, even if an incompressible Hookean solid should not be referred to as an incompressible fluid.

Figure 14 depicts the total mechanical pressure, which was calculated from

$$
p=-\frac{1}{3}\left(\sigma_{r r}+\sigma_{\vartheta \vartheta}+\sigma_{\varphi \varphi}\right),
$$

i.e., the combined action of gravitation and centrifugal acceleration. The equation was evaluated for $\vartheta=\pi / 2$ using (3-1) and Earth data. The same color code as before applies. However, as expected from our previous discussion, gravitation is dominant. In other words the plots look essentially the same for other values of $\vartheta$. Note the agreement with Figure 7 (right) for the case $v=0.5$. The curious 
cross-over point is visible again and the predicted pressure is well below the ones predicted by the PREM model. Clearly the calculation of the pressure according to (3-14) and (3-15) is formal and does not satisfy the boundary condition $p(r / R)=0$ unless the incompressibility condition $v=0.5$ is satisfied.

\section{Conclusions and outlook}

In this paper we have provided a somewhat comprehensive analysis of the flattening phenomenon. We started from its history, which is almost 400 years old. Then two constitutive models were discussed extensively. In the first model, the spinning celestial body was treated as an incompressible fluid and, in the second one, as a linear-elastic Hookean material. The corresponding boundary value problems were defined, and the local field equations were solved in closed form. Both models were numerically evaluated for terrestrial planets, gas giants, moons, and asteroids, as well as stars. The predictions for the corresponding flattening values were compared to experimental observations. Reasons for discrepancies were discussed. It is particularly noteworthy that the linear-elastic solution is not valid for larger telluric planets including Earth. A nonlinear solution for large deformations needs to be found in this case. This issue is extensively discussed in [Müller and Weiss 2016].

In most of our investigations a homogeneous mass density was a prerequisite. So far, an onion-layered type of Earth model for the flattening has neither been formulated nor analyzed. In principle the Hookean approach presented in Section 3 would allow one to study such a situation by adding certain transition conditions between the layers. In fact, it would even be possible to combine the linear-elastic and the fluid model by determining the coefficients of the Legendre series appropriately. However, such a solution would definitely involve a considerable amount of additional algebra and, in the end, most likely lead to rather cumbersome expressions. Therefore, it seems justified to perform a fully numerical analysis from the very beginning on, for example by discretizing the Earth using finite elements. This way not only the effects of a heterogeneous mass density distribution could be investigated, but also the impact of constitutive equations beyond linear elasticity. All of this is left to future investigations.

\section{Appendices}

In the following sections we present the derivation of some important formulae for the fluid as well as for the linear solid model. In fact, these results have been presented before in [Müller and Lofink 2014] but for the convenience of the reader they are compiled here once more. 


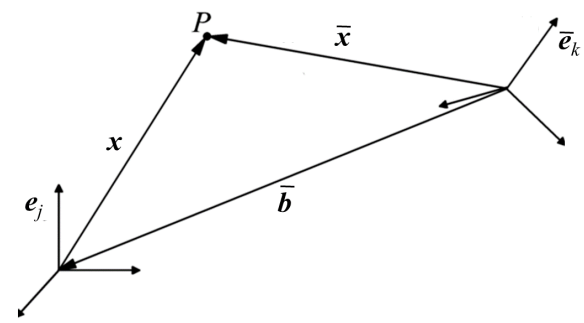

Figure 15. Notations used for Euclidean transformations.

\section{Appendix A: Derivation of pressure and flattening formulae for a rotating, self-gravitating body made of an incompressible fluid}

The following text is adapted in part from [Müller and Lofink 2014], which contained several typographical errors. The text arrangement was also revised for didactic reasons.

Recall the Euclidian transformation $\overline{\boldsymbol{x}}=\boldsymbol{Q} \cdot \boldsymbol{x}+\overline{\boldsymbol{b}}$ between an inertial system and a noninertial frame (identifiable by the bar with the Cartesian unit base $\overline{\boldsymbol{e}}_{i}$; see Figure 15), $\boldsymbol{Q}$ being the rotation tensor. The origins of the two systems are separated by the vector $\overline{\boldsymbol{b}}$. We may write for the relations between the velocities and the accelerations in both systems (see, e.g., [Müller 2014, p. 183])

$$
\begin{aligned}
\overline{\boldsymbol{x}}=\boldsymbol{Q} \cdot \boldsymbol{x}+\overline{\boldsymbol{b}} & \Rightarrow \quad \overline{\boldsymbol{v}}=\boldsymbol{Q} \cdot \boldsymbol{v}+\boldsymbol{\Omega} \cdot(\overline{\boldsymbol{x}}-\overline{\boldsymbol{b}})+\dot{\bar{b}} \\
\Longrightarrow & \overline{\boldsymbol{a}}=\boldsymbol{Q} \cdot \boldsymbol{a}+2 \boldsymbol{\Omega} \cdot(\overline{\boldsymbol{v}}-\dot{\bar{b}})+(\dot{\boldsymbol{\Omega}}-\boldsymbol{\Omega} \cdot \boldsymbol{\Omega}) \cdot(\overline{\boldsymbol{x}}-\overline{\boldsymbol{b}})+\ddot{\bar{b}},
\end{aligned}
$$

$\boldsymbol{\Omega}:=\dot{\boldsymbol{Q}} \cdot \boldsymbol{Q}^{\top}$ being the spin matrix. The angular velocity vector is given by $\boldsymbol{\omega}=-\frac{1}{2} \Omega_{i j} \overline{\boldsymbol{e}}_{i} \times \overline{\boldsymbol{e}}_{j}$.

Thus, the balance of momentum for the noninertial system reads (see, e.g., [Müller 2014, p. 199])

$$
\bar{\rho} \frac{\mathrm{d} \overline{\boldsymbol{v}}}{\mathrm{d} t}=\bar{\nabla} \cdot \overline{\boldsymbol{\sigma}}+\bar{\rho}(\overline{\boldsymbol{f}}+\overline{\boldsymbol{i}}),
$$

where the inertial accelerations were collected in

$$
\overline{\boldsymbol{i}}=2 \boldsymbol{\Omega} \cdot(\overline{\boldsymbol{v}}-\dot{\overline{\boldsymbol{b}}})+(\dot{\boldsymbol{\Omega}}-\boldsymbol{\Omega} \cdot \boldsymbol{\Omega}) \cdot(\overline{\boldsymbol{x}}-\overline{\boldsymbol{b}})+\ddot{\overline{\boldsymbol{b}}} .
$$

The Earth rotates at a constant angular speed, i.e., $\dot{\boldsymbol{\omega}}=\overline{\mathbf{0}}$, and we assume stationary conditions, i.e., $\overline{\boldsymbol{v}}=\overline{\mathbf{0}}$. Moreover, the origins of the two systems shall coincide, i.e., $\overline{\boldsymbol{b}}=\overline{\mathbf{0}}$. Then

$$
\bar{\nabla} \cdot \overline{\boldsymbol{\sigma}}+\bar{\rho}(\overline{\boldsymbol{f}}-\boldsymbol{\omega} \times(\boldsymbol{\omega} \times \overline{\boldsymbol{x}}))=\overline{\mathbf{0}} .
$$

For a fluid at rest (with respect to the noninertial frame) the stress tensor reduces to an isotropic pressure, i.e., $\overline{\boldsymbol{\sigma}}=-\bar{p} \overline{\boldsymbol{I}}$. Moreover, potentials can be used to obtain the 
gravitational as well as the centrifugal acceleration by differentiation with respect to position, i.e., $\overline{\boldsymbol{f}}=-\bar{\nabla} \bar{U}^{f}$ and $-\boldsymbol{\omega} \times(\boldsymbol{\omega} \times \overline{\boldsymbol{x}})=-\bar{\nabla} \bar{U}^{\omega}$. Finally we assume incompressibility, i.e., $\bar{\rho}=\bar{\rho}_{0}=$ const, and therefore,

$$
\bar{\nabla}\left[\bar{p}+\bar{\rho}_{0}\left(\bar{U}^{f}+\bar{U}^{\omega}\right)\right]=\overline{\mathbf{0}} .
$$

We use Cartesian coordinates in the noninertial frame (i.e., comoving ones, which explains the bar), such that $\omega, \omega_{0}=$ const, and consequently

$$
-\bar{\nabla} \bar{U}^{\omega}=-\boldsymbol{\omega} \times(\boldsymbol{\omega} \times \overline{\boldsymbol{x}})=\omega_{0}^{2}\left(\bar{x}_{1} \overline{\boldsymbol{e}}_{1}+\bar{x}_{2} \overline{\boldsymbol{e}}_{2}\right) \quad \Longrightarrow \quad \bar{U}^{\omega}=-\frac{1}{2} \omega_{0}^{2}\left(\bar{x}_{1}^{2}+\bar{x}_{2}^{2}\right) .
$$

In general, we may write for the gravitational potential at a point $\overline{\boldsymbol{x}}$ within an inhomogeneous material region $\bar{V}^{\prime}$ [Fitzpatrick 2011, p. 170]

$$
\bar{U}^{f}(\overline{\boldsymbol{x}})=-G \int_{\bar{V}^{\prime}} \frac{\bar{\rho}\left(\overline{\boldsymbol{x}}^{\prime}\right) \mathrm{d} \bar{V}^{\prime}}{\left|\overline{\boldsymbol{x}}-\overline{\boldsymbol{x}}^{\prime}\right|} .
$$

In order to solve the integral we have to specify the mass density within the body as well as its shape. For the case of a homogeneous ellipsoid with three different principal axes, $a_{i}$, (also known as MacLaurin ellipsoid in geodesy) the threedimensional integration can be reduced to one-dimensional integrals [Fitzpatrick 2016, p. 45] (Einstein's summation rule applies; $m$ denotes the total mass of the ellipsoid):

$$
\begin{array}{r}
\bar{U}^{f}(\overline{\boldsymbol{x}})=-\frac{3}{4} G m\left(\alpha_{0}-\alpha_{i} \bar{x}_{i}^{2}\right) \equiv-\pi G \bar{\rho}_{0} a_{1} a_{2} a_{3}\left(\alpha_{0}-\alpha_{i} \bar{x}_{i}^{2}\right), \quad \alpha_{0}=\int_{0}^{\infty} \frac{\mathrm{d} u}{\Delta}, \\
\alpha_{i}=\int_{0}^{\infty} \frac{\mathrm{d} u}{\left(a_{i}^{2}+u\right) \Delta}, \quad \Delta=\left(a_{1}^{2}+u\right)^{1 / 2}\left(a_{2}^{2}+u\right)^{1 / 2}\left(a_{3}^{2}+u\right)^{1 / 2} .
\end{array}
$$

Note that in this equation we anticipate the equilibrium shape of the spinning Earth: it is assumed to be a spheroid, i.e., an ellipsoid with two principal axes of equal length. With this in mind (A-5) can now be integrated, and the result is

$$
\bar{p}(\overline{\boldsymbol{x}})=\bar{p}(\overline{\mathbf{0}})-\frac{1}{2} \bar{\rho}_{0}\left[\left(\frac{3}{2} G m \alpha_{1}-\omega_{0}^{2}\right) \bar{x}_{1}^{2}+\left(\frac{3}{2} G m \alpha_{2}-\omega_{0}^{2}\right) \bar{x}_{2}^{2}+\frac{3}{2} G m \alpha_{3} \bar{x}_{3}^{2}\right] .
$$

This equation contains several unknowns, namely the central pressure $\bar{p}(\overline{\mathbf{0}})$ and the lengths of the principal axes, $a_{i}$. In fact, we must write them as functions of the mass of the spheroid, its angular speed, etc. In order to find out how, we concentrate on the outer periphery of the ellipsoid, which is described by the equation

$$
\frac{\bar{X}_{1}^{2}}{a_{1}^{2}}+\frac{\bar{X}_{2}^{2}}{a_{2}^{2}}+\frac{\bar{X}_{3}^{2}}{a_{3}^{2}}=1 .
$$

Note that we identify locations on the periphery by the vector $\overline{\boldsymbol{X}}$. Moreover, we assume that the atmospheric pressure acting on the Earth's surface can be neglected 
and is set equal to zero. Therefore, (A-9) leads to

$$
\frac{\bar{X}_{1}^{2}}{\left(\alpha_{1}-\frac{\omega_{0}^{2}}{\frac{3}{2} G m}\right)^{-1} \frac{4 \bar{p}(\overline{\mathbf{0}})}{3 \bar{\rho}_{0} G m}}+\frac{\bar{X}_{2}^{2}}{\left(\alpha_{2}-\frac{\omega_{0}^{2}}{\frac{3}{2} G m}\right)^{-1} \frac{4 \bar{p}(\overline{\mathbf{0}})}{3 \bar{\rho}_{0} G m}}+\frac{\bar{X}_{3}^{2}}{\alpha_{3}^{-1} \frac{4 \bar{p}(\overline{\mathbf{0}})}{3 \bar{\rho}_{0} G m}}=1,
$$

due to the requirement of continuity of the tractions of a surface at rest (with respect to the noninertial frame). Equations (A-10) and (A-11) must be satisfied simultaneously; hence,

$$
\frac{4 \bar{p}(\overline{\mathbf{0}})}{3 \bar{\rho}_{0} G m}=\left(\alpha_{1}-\frac{\omega_{0}^{2}}{\frac{3}{2} G m}\right) a_{1}^{2}=\left(\alpha_{2}-\frac{\omega_{0}^{2}}{\frac{3}{2} G m}\right) a_{2}^{2}=\alpha_{3} a_{3}^{2} .
$$

This leads after some algebraic manipulations to

$$
\left(a_{2}^{2}-a_{1}^{2}\right) \int_{0}^{\infty}\left[\frac{a_{1}^{2} a_{2}^{2}}{\left(a_{1}^{2}+u\right)\left(a_{2}^{2}+u\right)}-\frac{a_{3}^{2}}{\left(a_{3}^{2}+u\right)}\right] \frac{\mathrm{d} u}{\Delta}=0 .
$$

Therefore, the ellipsoid must be a spheroid, i.e., $a_{1}=a_{2} \equiv a$, as might be expected due to the rotation about a fixed axis and isotropy of space. It is interesting to note that there is experimental evidence which shows that this is only approximately true. Bretagnon et al. [1997] or Burša [1992] report that three principal moments of inertia are required to describe the observed precession rate of the Earth more accurately, $I_{11} \equiv A=0.329611083 \cdot m_{E} a_{E}^{2}, I_{22} \equiv B=0.329618344 \cdot m_{E} a_{E}^{2}$, and $I_{33} \equiv C=0.330697340 \cdot m_{E} a_{E}^{2}$, two of which are very similar. We conclude that the resistance to rotation about the polar axis is greatest, whereas resistance to rotation about the two equatorial principal axes is smaller and almost equal. Almost! $B$ is slightly larger than $A$. If we assume a homogeneous ellipsoid, such that $A=\frac{1}{5} m_{E}\left(b_{E}^{2}+c_{E}^{2}\right)$ and $B=\frac{1}{5} m_{E}\left(a_{E}^{2}+c_{E}^{2}\right)$, we conclude that $a_{E}$ should be slightly larger than $b_{E}$. However, for a homogeneous Earth ellipsoid we should also have $a_{E}=\sqrt{\left(5 /\left(2 m_{E}\right)\right)(B+C-A)}$, a relation which is not guaranteed by the numbers shown above. The reason is very simple: the mass distribution of the Earth is not homogeneous. Its core is much denser than its outside regions. This has an influence on its precession rate, a figure that was used to compute the numerical values for the principal moments of inertia. Thus, as common with all models, our simple assumption of a fluid, spheroidal Earth has its limits. It is worth pointing out that the earlier literature ignored differences between $A$ and $B$ due to insufficient accuracy of measurements at that time; see, e.g., [Munk and MacDonald 1960, Chapters 6 and 10]. It is also worth commenting that our implicit assumption according to which the principal axes of the real Earth point in the direction of the Earth's geographical pole and its equatorial regions is an approximation. Moreover, the polar or rather the third principal axis of the real Earth does not perfectly 
coincide with the direction of the angular velocity vector, a phenomenon which is known as the Earth's wobble. All of this underlines the limits of the assumption of a perfectly symmetric spheroid rotating about its polar axis.

On the other hand we obtain from (A-12)

$$
\frac{2 \omega_{0}^{2} a^{3}}{3 G m}=\frac{\left(1+2 \lambda^{2}\right) \arccos \lambda-3 \lambda \sqrt{1-\lambda^{2}}}{\left(1-\lambda^{2}\right)^{3 / 2}}, \quad \lambda=\frac{a_{3}}{a_{1}} \equiv \frac{c}{a},
$$

because the following integrals can now be solved in closed form:

$$
\begin{aligned}
\alpha_{0} & =\frac{1}{a} \int_{0}^{\infty} \frac{\mathrm{d} v}{(1+v)\left(\lambda^{2}+v\right)^{1 / 2}}=\frac{2}{a} \frac{\arccos \lambda}{\sqrt{1-\lambda^{2}}}, \quad v=\frac{u}{a^{2}}, \\
\alpha_{1} \equiv \alpha_{2} & =\frac{1}{a^{3}} \int_{0}^{\infty} \frac{\mathrm{d} v}{(1+v)^{2}\left(\lambda^{2}+v\right)^{1 / 2}}=\frac{1}{a^{3}} \frac{\lambda}{1-\lambda^{2}}\left[\frac{\arccos \lambda}{\lambda \sqrt{1-\lambda^{2}}}-1\right] \\
\alpha_{3} & =\frac{1}{a^{3}} \int_{0}^{\infty} \frac{\mathrm{d} v}{(1+v)\left(\lambda^{2}+v\right)^{3 / 2}}=\frac{1}{a^{3}} \frac{2\left(\sqrt{1-\lambda^{2}}-\lambda \arccos \lambda\right)}{\lambda\left(1-\lambda^{2}\right)^{3 / 2}} .
\end{aligned}
$$

We will now turn to (A-9) and compute the pressure function $\bar{p}(\overline{\boldsymbol{x}})$. This will later put us in a position to compare the result to expressions for the Hookean stresses. It is advisable to use generalized, comoving, spherical coordinates [Sokolov 1992] as follows:

$$
\begin{array}{r}
\bar{x}_{1}=a \bar{\xi}_{1} \cos \bar{\xi}_{2} \sin \bar{\xi}_{3}, \quad \bar{x}_{2}=a \bar{\xi}_{1} \sin \bar{\xi}_{2} \sin \bar{\xi}_{3}, \quad \bar{x}_{3}=c \bar{\xi}_{1} \cos \bar{\xi}_{3}, \\
\bar{\xi}_{1} \in[0,1], \quad \bar{\xi}_{2} \in[0,2 \pi], \quad \bar{\xi}_{3} \in[0, \pi]
\end{array}
$$

By combining (A-9), (A-12), and (A-15) we arrive at

$$
\bar{p}\left(\bar{\xi}_{1}\right)=\bar{p}(\overline{\mathbf{0}})\left(1-\bar{\xi}_{1}^{2}\right), \quad \bar{p}(\overline{\mathbf{0}})=\frac{3 \bar{\rho}_{0} G m}{2 a}\left(1-\frac{\lambda}{\sqrt{1-\lambda^{2}}} \arccos \lambda\right) \frac{\lambda}{1-\lambda^{2}} .
$$

\section{Appendix B: Derivation of the stress and displacement formulae for a linear-elastic solid model}

The presented solution follows a procedure outlined in the paper by Hiramatsu and Oka [1966]. It can also found in great detail in [Müller 2014, §9.6]. We start from the stationary local balance of momentum in a centrally comoving noninertial 
frame in spherical coordinates ignoring all explicit azimuthal dependences on $\varphi$ :

$$
\begin{aligned}
& \frac{\partial \sigma_{r r}}{\partial r}+\frac{1}{r} \frac{\partial \sigma_{r \vartheta}}{\partial \vartheta}+\frac{1}{r}\left(2 \sigma_{r r}-\sigma_{\vartheta \vartheta}-\sigma_{\varphi \varphi}+\sigma_{r \vartheta} \cot \vartheta\right) \\
& =\rho_{0}\left(\frac{G m_{0}}{R^{3}}-\omega_{0}^{2}\right) r+\rho_{0} \omega_{0}^{2} r \cos ^{2} \vartheta, \\
& \frac{\partial \sigma_{r \vartheta}}{\partial r}+\frac{1}{r} \frac{\partial \sigma_{\vartheta \vartheta}}{\partial \vartheta}+\frac{1}{r}\left[3 \sigma_{r \vartheta}+\left(\sigma_{\vartheta \vartheta}-\sigma_{\varphi \varphi}\right) \cot \vartheta\right]=-\rho_{0} \omega_{0}^{2} r \sin \vartheta \cos \vartheta, \\
& \frac{\partial \sigma_{r \varphi}}{\partial r}+\frac{1}{r} \frac{\partial \sigma_{\vartheta \varphi}}{\partial \vartheta}+\frac{1}{r}\left(3 \sigma_{r \varphi}+2 \sigma_{\vartheta \varphi} \cot \vartheta\right)=0 .
\end{aligned}
$$

For convenience we omit the bars introduced in the main text when denoting quantities of the noninertial frame, i.e., we do not write $\bar{\sigma}_{i j}$ or $\bar{u}_{i}$, for example, but $\sigma_{i j}$ and $u_{i}$ instead. The expressions on the right-hand side follow from the gravitational and centrifugal potentials shown in (A-6) and (A-8). A homogeneous mass density, $\rho_{0}$, was assumed throughout. This is in agreement with the conventions of linear elasticity where the forces are applied to the undeformed system, which in the present case is a sphere of radius $R$ and total mass $m_{0}=\frac{4}{3} \pi \rho_{0} R^{3}$. We now complement these equations by Hooke's law into which linear kinematic conditions for the displacements $\boldsymbol{u}$ are inserted:

$$
\begin{aligned}
\sigma_{r r} & =\lambda \Delta+2 \mu \frac{\partial u_{r}}{\partial r}, & \sigma_{\vartheta \vartheta} & =\lambda \Delta+\frac{2 \mu}{r}\left(\frac{\partial u_{\vartheta}}{\partial \vartheta}+u_{r}\right), \\
\sigma_{\varphi \varphi} & =\lambda \Delta+\frac{2 \mu}{r}\left(u_{r}+u_{\vartheta} \cot \vartheta\right), & \sigma_{r \vartheta} & =\mu\left[\frac{\partial u_{\vartheta}}{\partial r}-\frac{1}{r}\left(u_{\vartheta}-\frac{\partial u_{r}}{\partial \vartheta}\right)\right], \\
\sigma_{\vartheta \varphi} & =\frac{\mu}{r}\left(\frac{\partial u_{\varphi}}{\partial \vartheta}-u_{\varphi} \cot \vartheta\right), & \sigma_{r \varphi} & =\mu\left(\frac{\partial u_{\varphi}}{\partial r}-\frac{1}{r} u_{\varphi}\right)
\end{aligned}
$$

with the abbreviation

$$
\Delta=\frac{1}{r^{2} \sin \vartheta}\left[\frac{\partial}{\partial r}\left(r^{2} u_{r} \sin \vartheta\right)+\frac{\partial}{\partial \vartheta}\left(r u_{\vartheta} \sin \vartheta\right)\right] .
$$

Here $\lambda$ and $\mu$ denote Lamé's constants. It is now a matter of differentiation and algebra to show that (B-1) $)_{1,2}$ can be rewritten as

$$
\begin{aligned}
(\lambda+2 \mu) \frac{\partial \Delta}{\partial r}-\frac{2 \mu}{r} \frac{\partial \Omega}{\partial \vartheta}-\frac{2 \mu}{r} \Omega \cot \vartheta & =\rho_{0}\left(\frac{G m_{0}}{R^{3}}-\omega_{0}^{2}\right) r+\rho_{0} \omega_{0}^{2} r \cos ^{2} \vartheta, \\
(\lambda+2 \mu) \frac{1}{r} \frac{\partial \Delta}{\partial \vartheta}+2 \mu \frac{\partial \Omega}{\partial r}+2 \mu \frac{\Omega}{r} & =-\rho_{0} \omega_{0}^{2} r \sin \vartheta \cos \vartheta
\end{aligned}
$$

with an additional abbreviation

$$
2 \Omega=\frac{\partial u_{\vartheta}}{\partial r}+\frac{u_{\vartheta}}{\partial r}-\frac{1}{r} \frac{\partial u_{r}}{\partial \vartheta} .
$$


We will return to (B-1) 3 later. It will serve to determine $u_{\varphi}$ and is ignored for the time being. Cross-differentiation of (B-4) and mutual insertion leads to the decoupling of $\Delta$ and $\Omega$ :

$$
\begin{aligned}
\frac{\partial^{2} \Delta}{\partial r^{2}}+\frac{2}{r} \frac{\partial \Delta}{\partial r}+\frac{1}{r^{2}} \frac{\partial^{2} \Delta}{\partial \vartheta^{2}}+\frac{\cot \vartheta}{r^{2}} \frac{\partial \Delta}{\partial \vartheta} & =\frac{\rho_{0}\left(3 G m_{0} / R^{3}-2 \omega_{0}^{2}\right)}{\lambda+2 \mu}, \\
\frac{\partial^{2} \Omega}{\partial r^{2}}+\frac{2}{r} \frac{\partial \Omega}{\partial r}+\frac{1}{r^{2}} \frac{\partial^{2} \Omega}{\partial \vartheta^{2}}+\frac{\cot \vartheta}{r^{2}} \frac{\partial \Omega}{\partial \vartheta}-\frac{\Omega}{(r \sin \vartheta)^{2}} & =0 .
\end{aligned}
$$

These are equations of the Legendre type, and we may write their general solution in terms of Legendre series:

$$
\begin{aligned}
\Delta & =\frac{\rho_{0}\left(3 G m_{0} / R^{3}-2 \omega_{0}^{2}\right)}{6(\lambda+2 \mu)}+\sum_{n=0}^{\infty}\left(A_{n} r^{n}-\frac{B_{n}}{r^{n+1}}\right) P_{n}, \\
\Omega & =\sum_{n=0}^{\infty}\left(a_{n} r^{n}-\frac{b_{n}}{r^{n+1}}\right) \frac{\mathrm{d} P_{n}}{\mathrm{~d} \vartheta} .
\end{aligned}
$$

$P_{n}=P_{n}(\cos \vartheta)$ denotes the Legendre polynomial of the $n$-th degree. Note that in the formula for $\Delta$ the particular solution has been taken into account so that the inhomogeneity of the corresponding differential equation is covered. Moreover, the coefficients used to express $\Omega$ are related to those of $\Delta$ since (B-4) have to be observed. This leads to

$$
2 \Omega=-\frac{\lambda+2 \mu}{\mu} \sum_{n=0}^{\infty}\left(\frac{A_{n}}{n+1} r^{n}+\frac{B_{n}}{n} \frac{1}{r^{n+1}}\right) \frac{\mathrm{d} P_{n}}{\mathrm{~d} \vartheta}+\frac{\rho_{0} \omega_{0}^{2}}{9 \mu} r^{2} \frac{\mathrm{d} P_{2}}{\mathrm{~d} \vartheta} .
$$

These solutions help to find expressions for the two unknown displacements $u_{r}$ and $u_{\vartheta}$. To this end we use the definitions shown in (B-3) and (B-5) to obtain

$$
\begin{aligned}
\frac{\partial^{2} u_{\vartheta}}{\partial r^{2}}+\frac{1}{r^{2}} \frac{\partial^{2} u_{\vartheta}}{\partial \vartheta^{2}}+\frac{4}{r} \frac{\partial u_{\vartheta}}{\partial r}+\frac{\cot \vartheta}{r^{2}} \frac{\partial u_{\vartheta}}{\partial \vartheta}+\frac{1}{r^{2}} & \left(2-\frac{1}{\sin ^{2} \vartheta}\right) u_{\vartheta} \\
& =\frac{\partial(2 \Omega)}{\partial r}+\frac{1}{r} \frac{\partial \Delta}{\partial \vartheta}+\frac{3}{r}(2 \Omega) .
\end{aligned}
$$

By observing (B-7) and (B-8) we see that the solution to this differential equation is again of the Legendre type:

$$
\begin{array}{r}
u_{\vartheta}=\sum_{n=0}^{\infty}\left[-\frac{(n+3) \lambda+(n+5) \mu}{(n+1)(2 n+3) 2 \mu} A_{n} r^{n+1}-\frac{(n-2) \lambda+(n-4) \mu}{n(2 n-1) 2 \mu} \frac{B_{n}}{r^{n}}\right. \\
\left.+C_{n} r^{n-1}-\frac{D_{n}}{r^{n+2}}\right] \frac{\mathrm{d} P_{n}}{\mathrm{~d} \vartheta}+\frac{5 \rho_{0} \omega_{0}^{2}}{126 \mu} r^{3} \frac{\mathrm{d} P_{2}}{\mathrm{~d} \vartheta} .
\end{array}
$$


Now that we have found $u_{\vartheta}$ we can obtain the radial displacement by integration from (B-3) and (B-5). If we suppress rigid body translation the final result reads

$$
\begin{array}{r}
u_{r}=\sum_{n=0}^{\infty}\left[-\frac{n \lambda+(n-2) \mu}{(2 n+3) 2 \mu} A_{n} r^{n+1}+\frac{(n+1) \lambda+(n+3) \mu}{(2 n-1) 2 \mu} \frac{B_{n}}{r^{n}}+n C_{n} r^{n-1}\right. \\
\left.+(n+1) \frac{D_{n}}{r^{n+2}}\right] P_{n}+\frac{\rho_{0}\left(3 G m_{0} / R^{3}-2 \omega_{0}^{2}\right)}{30(\lambda+2 \mu)} r^{3}+\frac{\rho_{0} \omega_{0}^{2}}{21 \mu} r^{3} P_{2} .
\end{array}
$$

Finally we obtain $u_{\varphi}$ by combining (B-1) 3 and (B-2) $)_{5,6}$ :

$$
u_{\varphi}=\sum_{n=1}^{\infty}\left(E_{n} r^{n}+\frac{F_{n}}{r^{n+1}}\right) \frac{\mathrm{d} P_{n}}{\mathrm{~d} \vartheta} .
$$

Before we turn to expressions for the stresses we try to simplify the series as much as possible by evaluating the boundary conditions

$$
\begin{aligned}
\left.u_{r \text { and } \vartheta}\right|_{r=0}<\infty & \Longrightarrow B_{n}=0, n=1,2, \ldots, \quad D_{n}=0, n=0,1, \ldots, \\
\left.u_{r}\right|_{r=0}=0 & \Rightarrow B_{0}=0, \quad C_{1}=0, \\
\left.u_{\varphi}\right|_{r=0}<\infty & \Longrightarrow F_{n}=0, n=1,2, \ldots
\end{aligned}
$$

Note that $C_{0}$ and $F_{0}$ are irrelevant because of the prefactor $n$ and $\frac{\mathrm{d} P_{0}}{\mathrm{~d} \vartheta}=0$, respectively. Thus, according to (B-2) the stresses relevant for traction boundary conditions at the outer surface read

$$
\begin{aligned}
& \sigma_{r r}=\sum_{n=0}^{\infty}[-\left.\frac{\left(n^{2}-n-3\right) \lambda+(n+1)(n-2) \mu}{2 n+3} A_{n} r^{n}+n(n-1) 2 \mu C_{n} r^{n-2}\right] P_{n} \\
& \quad+\frac{5 \lambda+6 \mu}{10} \frac{\rho_{0}\left(G m_{0} / R^{3}\right)}{\lambda+2 \mu} r^{2}-\frac{5 \lambda+6 \mu}{15}+\frac{\rho_{0} \omega_{0}^{2}}{\lambda+2 \mu} r^{2}+\frac{2}{7} \rho_{0} \omega_{0}^{2} r^{2} P_{2}, \\
& \sigma_{r \vartheta}= \sum_{n=1}^{\infty}\left[-\frac{n(n+2) \lambda+\left(n^{2}+2 n-1\right) \mu}{(n+1)(2 n+3)} A_{n} r^{n}+2 \mu(n-1) C_{n} r^{n-2}\right] \frac{\mathrm{d} P_{n}}{\mathrm{~d} \vartheta} \\
& \quad+\frac{8}{63} \rho_{0} \omega_{0}^{2} r^{2} \frac{\mathrm{d} P_{2}}{\mathrm{~d} \vartheta} \\
& \sigma_{r \varphi}=\mu \sum_{n=1}^{\infty}(n-1) E_{n} r^{n-1} \frac{\mathrm{d} P_{n}}{\mathrm{~d} \vartheta} .
\end{aligned}
$$

The traction vector at the outer surface $r=R$ vanishes and

$$
\left.\sigma_{r \varphi}\right|_{r=R}=0 \quad \Longrightarrow \quad E_{n}=0, \quad n=2,3, \ldots,
$$

because of the linear independence of the polynomial expressions $\frac{\mathrm{d} P_{n}}{\mathrm{~d} \vartheta}$. We conclude that

$$
\sigma_{r \varphi} \equiv 0, \quad u_{\varphi} \equiv 0
$$


Moreover,

$$
\begin{aligned}
& \left.\sigma_{r \vartheta}\right|_{r=R}=0 \Longrightarrow \\
& \begin{cases}-\frac{n(n+2) \lambda+\left(n^{2}+2 n-1\right) \mu}{(n+1)(2 n+3)} R^{n} A_{n}+2 \mu(n-1) R^{n-2} C_{n}=0, & n \neq 2, \\
-\frac{8 \lambda+7 \mu}{21} R^{2} A_{2}+2 \mu C_{2}+\frac{8}{63} \rho_{0} \omega_{0}^{2} R^{2}=0, & n=2 .\end{cases}
\end{aligned}
$$

And finally

$$
\begin{aligned}
& \left.\sigma_{r r}\right|_{r=R}=0 \Longrightarrow \\
& \begin{cases}\frac{5 \lambda+6 \mu}{10} \frac{\rho_{0}\left(G m_{0} / R^{3}\right)}{\lambda+2 \mu} R^{2}-\frac{5 \lambda+6 \mu}{15} \frac{\rho_{0} \omega_{0}^{2}}{\lambda+2 \mu} R^{2}+\frac{3 \lambda+2 \mu}{3} A_{0}=0, \\
n=0, \\
\frac{3 \lambda+2 \mu}{7} R A_{1}=0 \Rightarrow A_{1}=0, & n=1, \\
\frac{2}{7} \rho_{0} \omega_{0}^{2} R^{2}+\frac{\lambda}{7} A_{2} R^{2}+4 \mu C_{2}=0, & n=2, \\
-\frac{\left(n^{2}-n-3\right) \lambda+(n+1)(n-2) \mu}{(2 n+3)} R^{n} A_{n}+2 \mu n(n-1) R^{n-2} C_{n} & =0, \\
& n>2 .\end{cases}
\end{aligned}
$$

We are now in a position to determine all remaining coefficients:

$$
\begin{aligned}
& A_{0}=-\frac{3}{10} \frac{5 \lambda+6 \mu}{3 \lambda+2 \mu} \frac{\rho_{0}\left(G m_{0} / R^{3}\right)}{\lambda+2 \mu} R^{2}+\frac{1}{5} \frac{5 \lambda+6 \mu}{3 \lambda+2 \mu} \frac{\rho_{0} \omega_{0}^{2}}{\lambda+2 \mu} R^{2}, \\
& A_{1}=0, \quad A_{2}=-\frac{2}{3} \frac{\rho_{0} \omega_{0}^{2}}{19 \lambda+14 \mu}, \quad A_{n}=0, \quad n=3,4, \ldots, \\
& C_{2}=-\frac{4 \lambda+3 \mu}{19 \lambda+14 \mu} \frac{\rho_{0} \omega_{0}^{2}}{3 \mu} R^{2}, \quad C_{n}=0, \quad n=3,4, \ldots
\end{aligned}
$$

This result leads directly to the expressions for the stresses and displacements presented in (3-1) and (3-2).

\section{References}

[Anderson et al. 2001a] J. D. Anderson, R. A. Jacobson, E. L. Lau, W. B. Moore, O. Olsen, G. Schubert, P. C. Thomas, and the Galileo Gravity Science Team, "Shape, mean radius, gravity field and interior structure of Ganymede", B. Am. Astro. Soc. 33 (2001), 1101.

[Anderson et al. 2001b] J. D. Anderson, R. A. Jacobson, T. P. McElrath, W. B. Moore, G. Schubert, and P. C. Thomas, "Shape, mean radius, gravity field, and interior structure of Callisto", Icarus 153:1 (2001), 157-161.

[Blanch et al. 94] G. Blanch, A. N. Lowan, R. E. Marshak, and H. A. Bethe, "The internal temperaturedensity distribution of the Sun", Astrophys. J. 94 (94), 37-45.

[Bretagnon et al. 1997] P. Bretagnon, P. Rocher, and J. L. Simon, "Theory of the rotation of the rigid Earth", Astron. Astrophys. 319:1 (1997), 305-317.

[Bridgman 1958] P. W. Bridgman, The logic of modern physics, Macmillan, 1958. 
[Burša 1992] M. Burša, "Current estimates of the Earth's principal moments of inertia", Stud. Geophys. Geod. 36:2 (1992), 109-114.

[Carrington 1859] R. C. Carrington, "On certain phenomena in the motions of solar spots", Mon. Not. R. Astron. Soc. 19:3 (1859), 81-84.

[Chandrasekhar 1933] S. Chandrasekhar, "The equilibrium of distorted polytropes, I: The rotational problem”, Mon. Not. R. Astron. Soc. 93:5 (1933), 390-406.

[Chandrasekhar 1995] S. Chandrasekhar, Newton's Principia for the common reader, Clarendon, 1995.

[Dehant et al. 2003] V. Dehant, K. C. Creager, S.-I. Karato, and S. Zatman (editors), Earth's core: dynamics, structure, rotation, Geodynamic Series 31, American Geophysical Union, 2003.

[Dziewonski and Anderson 1981] A. M. Dziewonski and D. L. Anderson, "Preliminary reference Earth model", Phys. Earth Planet. In. 25:4 (1981), 297-356.

[Fitzpatrick 2011] R. Fitzpatrick, "Newtonian dynamics", lecture notes, University of Texas at Austin, 2011, available at http://farside.ph.utexas.edu/teaching/336k/Newton.pdf.

[Fitzpatrick 2016] R. Fitzpatrick, "Fluid mechanics", lecture notes, University of Texas at Austin, 2016, available at http://farside.ph.utexas.edu/teaching/336L/Fluid/.

[Franco et al. 2000] L. M. Franco, B. Link, and R. I. Epstein, "Quaking neutron stars”, Astrophys. J. 543:2 (2000), 987-994.

[Galilei 1613] G. Galilei, Istoria e dimostrazioni intorno alle macchie solari e loro accidenti, Mascardi, 1613.

[Galilei and Scheiner 2010] G. Galilei and C. Scheiner, On sunspots, University of Chicago, 2010.

[Godier and Rozelot 1999] S. Godier and J.-P. Rozelot, "Quadrupole moment of the Sun: gravitational and rotational potentials", Astron. Astrophys. 350:1 (1999), 310-317.

[Godier and Rozelot 2000] S. Godier and J.-P. Rozelot, "The solar oblateness and its relationship with the structure of the tachocline and of the Sun's subsurface", Astron. Astrophys. 355:1 (2000), $365-374$.

[Hiramatsu and Oka 1966] Y. Hiramatsu and Y. Oka, "Determination of the tensile strength of rock by a compression test of an irregular test piece", Int. J. Rock Mech. Min. Sci. 3:2 (1966), 89-90.

[Horedt 2004] G. P. Horedt, Polytropes: applications in astrophysics and related fields, Astrophysics and Space Science Library 306, Kluwer, 2004.

[Klein and Sommerfeld 2012] F. Klein and A. Sommerfeld, The theory of the top, vol. III: Perturbations, astronomical and geophysical applications, Springer, 2012.

[Kosovichev 1998] A. G. Kosovichev, "Seismic observation of solar tachocline", pp. 97-98 in Sounding solar and stellar interiors (Nice, 1997), edited by J. Provost and F. X. Schmider, International Astronomical Union Symposia 181, Kluwer, 1998.

[Lee et al. 2002] W. H. K. Lee, H. Kanamori, P. C. Jennings, and C. Kisslinger (editors), International handbook of earthquake and engineering seismology, part A, International Geophysics 81, Academic, 2002.

[Love 1911] A. E. H. Love, Some problems of geodynamics, Cambridge University, 1911.

[MacLaurin 1742] C. MacLaurin, A treatise of fluxions in two books, vol. I, Ruddimans, 1742.

[Miles and Ramsey 1952] B. Miles and W. H. Ramsey, "On the internal structures of Jupiter and Saturn", Mon. Not. R. Astron. Soc. 112:2 (1952), 234-243.

[Milne 1923] E. A. Milne, "The equilibrium of a rotating star", Mon. Not. R. Astron. Soc. 83:3 (1923), 118-147. 
[Müller 2014] W. H. Müller, An expedition to continuum theory, Solid Mechanics and its Applications 210, Springer, 2014.

[Müller and Lofink 2014] W. H. Müller and P. Lofink, "The movement of the earth: modelling the flattening parameter", Lect. Notes TICMI 15 (2014), 40.

[Müller and Weiss 2016] W. H. Müller and W. Weiss, The state of deformation in earthlike selfgravitating objects, Springer, 2016.

[Munk and MacDonald 1960] W. H. Munk and G. J. F. MacDonald, The rotation of the Earth: A geophysical discussion, Cambridge University, 1960.

[NASA 2017] NASA, "Pioneer 11 image of Saturn and its moon Titan", 2017, available at http:// www.nasa.gov/multimedia/imagegallery/image_feature_2483.html.

[Newton 1972] I. Newton, Philosophiae naturalis principia mathematica, 3rd ed., Harvard University, 1972.

[Newton 1999] I. Newton, The Principia: mathematical principles of natural philosophy, University of California Press, 1999.

[Poincaré 1885] H. Poincaré, "Sur l'équilibre d'une masse fluide animée d'un mouvement de rotation", Acta Math. 7:1 (1885), 259-380.

[Rozelot and Neiner 2011] J.-P. Rozelot and C. Neiner (editors), The pulsations of the Sun and the stars, Lecture Notes in Physics 832, Springer, 2011.

[Rozelot et al. 2011] J.-P. Rozelot, C. Damiani, A. Kilcik, B. Tayoglu, and S. Lefebvre, "Unveiling stellar cores and multipole moments via their flattening", Chapter 5, pp. 161-181 in The pulsations of the Sun and the stars, edited by J.-P. Rozelot and C. Neiner, Lecture Notes in Physics 832, Springer, 2011.

[Sokolov 1992] D. D. Sokolov, "Spherical coordinates", pp. 435-436 in Encyclopaedia of mathematics, vol. 8, edited by M. Hazewinkel, Kluwer, 1992.

[Stacey and Davis 2008] F. D. Stacey and P. M. Davis, Physics of the Earth, 4th ed., Cambridge University, 2008.

[Thomas et al. 1998] P. C. Thomas, M. E. Davies, T. R. Colvin, J. Oberst, P. Schuster, G. Neukum, M. H. Carr, A. McEwen, G. Schubert, M. J. S. Belton, and the Galileo Imaging Team, "The shape of Io from Galileo limb measurements”, Icarus 135:1 (1998), 175-180.

[Thomson 1863] W. Thomson, "On the rigidity of the Earth", Phil. Tr. R. Soc. Lond. 153:2 (1863), $573-582$.

[Thomson and Tait 1912] W. Thomson and P. Tait, Treatise on natural philosophy, part II, Cambridge University, 1912.

[Turcotte and Pflugrath 1984] D. L. Turcotte and J. C. Pflugrath, "Was the early Earth completely molten?", pp. 870-871 in Lunar and Planetary Science Conference (Saint Petersburg, 1984), vol. 15, Russian Academy of Sciences, 1984.

[Turcotte and Schubert 2002] D. Turcotte and G. Schubert, Geodynamics, 2nd ed., Cambridge University, 2002.

[Van Helden 1995] A. Van Helden, "The Galileo Project: sunspots", 1995, available at http:// galileo.rice.edu/sci/observations/sunspots.html.

[Van Hoolst et al. 2008] T. Van Hoolst, N. Rambaux, O. Karatekin, V. Dehant, and A. Rivoldini, "The librations, shape, and icy shell of Europa", Icarus 195:1 (2008), 386-399.

[Wikipedia 2015a] "Callisto (moon)", Wikipedia entry, 2015, available at http://en.wikipedia.org/ wiki/Callisto_(moon). 
[Wikipedia 2015b] "Europa (moon)", Wikipedia entry, 2015, available at http://en.wikipedia.org/ wiki/Europa_(moon).

[Wikipedia 2015c] "Ganymede (moon)", Wikipedia entry, 2015, available at http://en.wikipedia.org/ wiki/Ganymede_(moon).

[Wikipedia 2015d] "Io (moon)", Wikipedia entry, 2015, available at http://en.wikipedia.org/wiki/ Io_(moon).

[Wikipedia 2015e] "Moon", Wikipedia entry, 2015, available at http://en.wikipedia.org/wiki/Moon.

[Wikipedia 2015f] “Titan (moon)”, Wikipedia entry, 2015, available at http://en.wikipedia.org/wiki/ Titan_(moon).

[Wikipedia 2016a] "Earth”, Wikipedia entry, 2016, available at http://en.wikipedia.org/wiki/Earth.

[Wikipedia 2016b] "Jupiter", Wikipedia entry, 2016, available at http://en.wikipedia.org/wiki/Jupiter.

[Wikipedia 2016c] “Sidereal time”, Wikipedia entry, 2016, available at http://en.wikipedia.org/wiki/ Sidereal_time.

[Williams 2017] D. R. Williams, "Planetary fact sheet: metric", fact sheet, NASA, 2017, available at http://nssdc.gsfc.nasa.gov/planetary/factsheet.

[Zebker et al. 2009] H. A. Zebker, B. Stiles, S. Hensley, R. Lorenz, R. L. Kirk, and J. Lunine, "Size and shape of Saturn's moon Titan", Science 324:5929 (2009), 921-923.

Received 31 May 2017. Revised 23 Oct 2017. Accepted 2 Dec 2017.

WOLFGANG H. MÜLLER: whmueller1000@gmail.com

Institut für Mechanik, Technische Universität Berlin, Berlin, Germany 